\title{
First light of the VLT planet finder SPHERE
}

\section{New spectrophotometry and astrometry of the HR 8799 exoplanetary system $^{\star}, \star \star$}

\author{
A. Zurlo ${ }^{1,2,3,4}$, A. Vigan ${ }^{3,5}$, R. Galicher ${ }^{6}$, A.-L. Maire ${ }^{4}$, D. Mesa $^{4}$, R. Gratton ${ }^{4}$, G. Chauvin ${ }^{7,8}$, M. Kasper ${ }^{9,7,8}$,
} C. Moutou ${ }^{3}$, M. Bonnefoy ${ }^{7,8}$, S. Desidera ${ }^{4}$, L. Abe ${ }^{10}$, D. Apai ${ }^{11,12, \star \star \star}$, A. Baruffolo ${ }^{4}$, P. Baudoz ${ }^{6}$, J. Baudrand ${ }^{6}$, J.-L. Beuzit ${ }^{7,8}$, P. Blancard ${ }^{3}$, A. Boccaletti ${ }^{6}$, F. Cantalloube ${ }^{7,8}$, M. Carle ${ }^{3}$, E. Cascone ${ }^{13}$, J. Charton ${ }^{8}$, R. U. Claudi ${ }^{4}$, A. Costille ${ }^{3}$, V. de Caprio $^{13}$, K. Dohlen ${ }^{3}$, C. Dominik ${ }^{14}$, D. Fantinel ${ }^{4}$, P. Feautrier ${ }^{8}$, M. Feldt ${ }^{15}$, T. Fusco ${ }^{3,16}$, P. Gigan ${ }^{6}$, J. H. Girard ${ }^{5,7,8}$, D. Gisler ${ }^{17}$, L. Gluck ${ }^{7,8}$, C. Gry ${ }^{3}$, T. Henning ${ }^{15}$, E. Hugot ${ }^{3}$, M. Janson ${ }^{18,15}$, M. Jaquet ${ }^{3}$, A.-M. Lagrange ${ }^{7,8}$, M. Langlois ${ }^{19,3}$, M. Llored ${ }^{3}$, F. Madec ${ }^{3}$, Y. Magnard ${ }^{8}$, P. Martinez ${ }^{10}$, D. Maurel ${ }^{8}$, D. Mawet ${ }^{20}$, M. R. Meyer ${ }^{21}$, J. Milli ${ }^{5,7,8}$, O. Moeller-Nilsson ${ }^{15}$, D. Mouillet ${ }^{7,8}$, A. Origné ${ }^{3}$, A. Pavlov ${ }^{15}$, C. Petit ${ }^{16}$, P. Puget ${ }^{8}$, S. P. Quanz ${ }^{21}$, P. Rabou ${ }^{8}$, J. Ramos ${ }^{15}$, G. Rousset ${ }^{6}$, A. Roux ${ }^{8}$, B. Salasnich ${ }^{4}$, G. Salter ${ }^{3}$, J.-F. Sauvage ${ }^{3,16}$, H. M. Schmid ${ }^{21}$, C. Soenke ${ }^{5}$, E. Stadler ${ }^{8}$, M. Suarez ${ }^{5}$, M. Turatto ${ }^{4}$, S. Udry ${ }^{22}$, F. Vakili ${ }^{10}$, Z. Wahhaj ${ }^{5}$, F. Wildi ${ }^{22}$, and J. Antichi ${ }^{23}$

(Affiliations can be found after the references)

Received 25 June 2015 / Accepted 2 November 2015

\section{ABSTRACT}

Context. The planetary system discovered around the young A-type HR 8799 provides a unique laboratory to: a) test planet formation theories; b) probe the diversity of system architectures at these separations, and c) perform comparative (exo)planetology.

Aims. We present and exploit new near-infrared images and integral-field spectra of the four gas giants surrounding HR 8799 obtained with SPHERE, the new planet finder instrument at the Very Large Telescope, during the commissioning and science verification phase of the instrument (July-December 2014). With these new data, we contribute to completing the spectral energy distribution (SED) of these bodies in the 1.0-2.5 $\mu \mathrm{m}$ range. We also provide new astrometric data, in particular for planet e, to further constrain the orbits.

Methods. We used the infrared dual-band imager and spectrograph (IRDIS) subsystem to obtain pupil-stabilized, dual-band $H 2 H 3$ (1.593 $\mu$ m, $1.667 \mu \mathrm{m}), K 1 K 2(2.110 \mu \mathrm{m}, 2.251 \mu \mathrm{m})$, and broadband $J(1.245 \mu \mathrm{m})$ images of the four planets. IRDIS was operated in parallel with the integral field spectrograph (IFS) of SPHERE to collect low-resolution $(R \sim 30)$, near-infrared $(0.94-1.64 \mu \mathrm{m})$ spectra of the two innermost planets HR $8799 \mathrm{~d}$ and e. The data were reduced with dedicated algorithms, such as the Karhunen-Loève image projection (KLIP), to reveal the planets. We used the so-called negative planets injection technique to extract their photometry, spectra, and measure their positions. We illustrate the astrometric performance of SPHERE through sample orbital fits compatible with SPHERE and literature data.

Results. We demonstrated the ability of SPHERE to detect and characterize planets in this kind of systems, providing spectra and photometry of its components. The spectra improve upon the signal-to-noise ratio of previously obtained data and increase the spectral coverage down to the $Y$ band. In addition, we provide the first detection of planet e in the $J$ band. Astrometric positions for planets HR 8799 bcde are reported for the epochs of July, August, and December 2014. We measured the photometric values in $J, H 2 H 3, K 1 K 2$ bands for the four planets with a mean accuracy of $0.13 \mathrm{mag}$. We found upper limit constraints on the mass of a possible planet f of 3-7 $M_{\text {Jup }}$. Our new measurements are more consistent with the two inner planets $d$ and e being in a 2d:1e or 3d:2e resonance. The spectra of HR $8799 \mathrm{~d}$ and e are well matched by those of L6-8 field dwarfs. However, the SEDs of these objects are redder than field $\mathrm{L}$ dwarfs longward of $1.6 \mu \mathrm{m}$.

Key words. techniques: image processing - astrometry - techniques: high angular resolution - techniques: spectroscopic - methods: data analysis

\section{Introduction}

The intriguing planetary system around HR 8799 is one of the most interesting objects in the field of extrasolar planets. Four giant planets have been detected (HR 8799 bcde; Marois et al. 2008, 2010b) as well as a double debris belt (Su et al. 2009; Hughes et al. 2011; Matthews et al. 2014).

* Based on observations collected at the European Southern Observatory (ESO), Chile, during the commissioning of the SPHERE instrument and during the science verification (program number 60.A-9352(A)).

$\star \star$ Spectra of planets are only available at the CDS via anonymous ftp to cdsarc.u-strasbg.fr $(130.79 .128 .5)$ or via

http://cdsarc.u-strasbg.fr/viz-bin/qcat?J/A+A/587/A57

$\star \star \star$ Earth in Other Solar Systems Team.
HR 8799 is a $\gamma$ Dor-type variable star (Gray \& Kaye 1999) with $\lambda$ Boo-like abundance patterns. Its age has been estimated to be 20-160 Myr (Cowley et al. 1969; Moór et al. 2006; Marois et al. 2008; Hinz et al. 2010; Zuckerman et al. 2011; Baines et al. 2012), and the distance of the system is $39.4 \pm 1.0 \mathrm{pc}$ (van Leeuwen 2007). This system is a benchmark for the study of young, gaseous giant planets, their formation and evolution. The planets have relative low contrast $(\Delta H \sim 12 \mathrm{mag})$ and are separated from their host star (15-70 AU) such that they are relatively easy to detect with the new generation of high-contrast imagers utilizing optimized image processing techniques. For an assumed age of $30 \mathrm{Myr}$ (Columba association), the measured luminosities of the planets suggest masses around 5-7 $M_{\mathrm{Jup}}$ (Marois et al. 2010b; Currie et al. 2011; Sudol \& Haghighipour 2012), in agreement with the upper limits set by dynamical 
stability studies of the system (Goździewski \& Migaszewski 2014).

So far, studies of the atmospheres of HR 8799 bcde were conducted using mostly photometric measurements in the near infrared. The system has been detected by several instruments, providing broadband photometry and/or astrometry in $z, J, H, K$, $L, M$ filters (Marois et al. 2008, 2010b; Lafrenière et al. 2009; Hinz et al. 2010; Janson et al. 2010; Serabyn et al. 2010; Currie et al. 2011; Hinkley et al. 2011a; Galicher et al. 2011; Soummer et al. 2011; Skemer et al. 2012, 2014; Esposito et al. 2013). A new generation of high-contrast imaging instruments such as SPHERE, GPI, ScEXAO+CHARIS, Project 1640 (Beuzit et al. 2008; Macintosh et al. 2014; McElwain et al. 2012; Hinkley et al. 2011b) can now provide the spectra of these objects, enabling deeper study of their chemistry and physical properties. Spectra of the companions have been obtained in the $J H$ band for planets b, c, d, and e by Project 1640 at Palomar (Oppenheimer et al. 2013), in the $H$ and $K$ bands using Keck/OSIRIS (Larkin et al. 2006) for planets b (Barman et al. 2011, 2015; Bowler et al. 2010) and c (Konopacky et al. 2013), exploiting GPI for planets c and d (Ingraham et al. 2014), and in 3.88-4.10 $\mu \mathrm{m}$ with VLT/NACO for planet c (Janson et al. 2010).

State-of-the-art analysis of the atmospheres presents a complex picture of the objects of this system and ad hoc models are needed to fit the spectrophotometric data. The planets around HR 8799 show nonequilibrium chemistry in the atmospheres and they appear dustier than field objects of the same effective temperature (Currie et al. 2011). The fact that HR 8799 bcde are brighter at $3.3 \mu \mathrm{m}$ than predicted by the equilibrium chemistry models optimized for old brown dwarfs suggests that the upper layers of their atmosphere are not as opaque as expected because of the lack of $\mathrm{CH}_{4}$ (Skemer et al. 2012). To reconcile the discrepancies between models and data, a patchy cloud coverage has been suggested by Marois et al. (2008), Currie et al. (2011), Skemer et al. (2012), Marley et al. (2012), and Morley et al. (2012). Madhusudhan et al. (2011) proposed a set of models that are intermediate between two sets of models; one where the physical extent of the clouds is truncated at a given altitude, and a second model where clouds extend all the way to the top of the atmosphere. The fact that we need thick clouds and nonequilibrium chemistry to fit the observables could be related to the low surface gravities of the planets around HR 8799 (Madhusudhan et al. 2011; Marley et al. 2012). This patchy cloud hypothesis is fully consistent with the high-precision rotational phase mapping studies of field brown dwarfs with spectra similar to HR 8799 b, demonstrating heterogeneous clouds (warm thin and cool thick clouds) for several objects (Apai et al. 2013; Buenzli et al. 2015).

In this paper, we present new data obtained with VLT/SPHERE of the objects in the system. We obtain photometry and astrometry in $J, H$, and $K$ bands for the four planets and extract $Y H$ band spectra for planets d and e. We then provide an updated interpretation of the complex atmospheres of the HR 8799 planets, taking advantage of the exquisite quality of the data obtained.

The outline of the paper is as follow: in Sect. 2 we present SPHERE near-infrared observations of the system around HR 8799 during the instrument commissioning and science verification runs in July, August, and December 2014; in Sect. 3 we describe the reduction methods applied and the results that we obtained. In Sect. 4 we present the astrometric fitting for the four planets of HR 8799. In Sect. 5 we present a first comparison with data from objects of comparable temperature, while a more
Table 1. IRDIS filters used during the observations and their resolutions.

\begin{tabular}{lcc}
\hline \hline Filter & Wavelength $(\mu \mathrm{m})$ & Resolution \\
\hline BB_J & 1.245 & 5 \\
BB_H & 1.625 & 6 \\
$H 2$ & 1.593 & 30 \\
$H 3$ & 1.667 & 30 \\
$K 1$ & 2.110 & 20 \\
$K 2$ & 2.251 & 20 \\
\hline
\end{tabular}

detailed analysis is found in Bonnefoy et al. (2016). We give the conclusions in Sect. 6.

\section{Observations}

We observed HR 8799 over three different nights during the SPHERE commissioning runs and four different nights during science verification. The SPHERE planet-finder instrument installed at the VLT (Beuzit et al. 2008) is a highly specialized instrument dedicated to high-contrast imaging. It was built by a large consortium of European laboratories. It is equipped with an extreme adaptive optics system, SAXO (Fusco et al. 2006; Petit et al. 2014), with a $41 \times 41$ actuators wavefront control, pupil stabilization, differential tip-tilt control, and it also employs stress polished toric mirrors for beam transportation (Hugot et al. 2012). The SPHERE instrument is equipped with several coronagraphic devices for stellar diffraction suppression, including apodized Lyot coronagraphs (Soummer 2005) and achromatic four-quadrants phase masks (Boccaletti et al. 2008). The instrument has three science subsystems: the infrared dual-band imager and spectrograph (IRDIS; Dohlen et al. 2008), an integral field spectrograph (IFS; Claudi et al. 2008) and the Zimpol rapid-switching imaging polarimeter (ZIMPOL; Thalmann et al. 2008).

Two sequences of data were acquired with the integral-field spectrometer IFS (Claudi et al. 2008) and the dual-band imager IRDIS (Dohlen et al. 2008) in dual-band imaging mode (Vigan et al. 2010) working in parallel. The last sequence was taken with IRDIS alone in the broadband imaging mode with the $J$ band filter. Finally, a broadband imaging sequence in $H$ band was taken during the science verification (SV) phase. Data in different spectral ranges were obtained in the following configurations:

- The first sequence was taken on July, 13th 2014 in IRDIFS mode (IRDIS in dual-band imaging mode using the $H 2 H 3$ filters, and IFS in the $Y J$ mode). The seeing was variable between $0.8-1.0^{\prime \prime}$. Sixteen datacubes were acquired, with a total exposure time of $43 \mathrm{~min}$. The total field of view (FoV) rotation during the observation was $18.1^{\circ}$.

- An IRDIFS_EXT sequence (IRDIS in dual-band imaging mode with the $K 1 K 2$ filters, and IFS in the $Y H$ mode) was taken on August, 12th 2014 with good seeing conditions. Thirty-two datacubes were acquired for each instrument with a total exposure time of nearly two hours. The total field rotation was $33.8^{\circ}$.

- An IRDIS broadband $J$ sequence was taken on August, 14th 2014. Thirty-two datacubes were acquired for an integration time of $\sim 1.4 \mathrm{~h}$. The total FoV rotation was $31.2^{\circ}$.

- Four IRDIS broadband $H$ sequences were taken, between December, 4th to 8th 2014, during SV. Integration times for each data set were around $30 \mathrm{~min}$, and field rotations were around $8^{\circ}$.

IRDIS filters and resolutions are listed in Table 1. The observations are summarized in Table 2. 
Table 2. Observations of HR 8799 during SPHERE commissioning and science verification runs.

\begin{tabular}{lcccccc}
\hline \hline UT date & IRDIS filter & IFS Band & IRDIS DIT $^{a} \times$ NDIT $^{b}$ & IFS DIT $\times$ NDIT & N. of datacubes & FoV rotation \\
\hline $2014-07-13$ & $H 2 H 3$ & $Y J$ & $4 \times 40$ & $8 \times 20$ & 16 & $18.1^{\circ}$ \\
$2014-08-12$ & K1K2 & $Y H$ & $(30+35) \times 6$ & $60 \times 3+100 \times 2$ & $16+16$ & $33.8^{\circ}$ \\
$2014-08-14$ & BB_J & - & $16 \times 10$ & - & 32 & $31.2^{\circ}$ \\
$2014-12-04$ & BB_H & - & $8 \times 218$ & - & 1 & $8.7^{\circ}$ \\
$2014-12-05$ & BB_H & - & $8 \times 218$ & - & 1 & $8.7^{\circ}$ \\
$2014-12-06$ & BB_H & - & $8 \times 218$ & - & 1 & $8.5^{\circ}$ \\
$2014-12-08$ & BB_H & - & $8 \times 218$ & 1 & $7.9^{\circ}$ \\
\hline
\end{tabular}

Notes. ${ }^{(a)}$ Detector Integration Time. ${ }^{(b)}$ Number of frames per dithering position.

All the sequences were taken with the same configuration of the apodized Lyot coronagraph (Carbillet et al. 2011), which includes a focal plane mask with a diameter of 185 mas and an inner working angle (IWA) of 0.'09.

The commissioning sequences were taken with the following observing strategy:

- A star centered frame, which is a coronagraphic image of the star with four satellite spots symmetric with respect to the central star, was acquired at the beginning and at the end of each observation by introducing a periodic modulation on the deformable mirror. The purpose of this observation is to accurately determine the star position for frame registering before the application of angular differential image (ADI) processing (Marois et al. 2006a) and for derivation of the astrometry for the detected companions relative to the star (Marois et al. 2006b; Sivaramakrishnan \& Oppenheimer 2006). The satellite spots have high $\mathrm{S} / \mathrm{N}(\sim 50)$, which permits a good estimation of the center with milliarcsecond precision.

- Nonsaturated images of the star shifted from the central axis using a tip-tilt mirror were taken at the beginning and at the end of the observations with a neutral density filter, ND2.0, with a transmission $\sim 1 \%$ of the total bandwidth, to calibrate the flux.

- Coronagraphic images were taken with a $4 \times 4$ dithering pattern for IRDIS. These images were obtained in pupil-stabilized mode to take advantage of the angular ADI technique.

- At the end of sequence six, sky background images were obtained with the same exposure time for the coronagraphic and unsaturated point spread function (PSF) images.

The broadband $H$ sequences during SV were acquired with the four satellite spots permanently applied. All the other calibrations described in Sects. 3.2 and 3.3 were acquired during the next morning as part of the instrument calibration plan.

\section{Data reduction, photometry and astrometry}

\subsection{Astrometric calibration}

During each run of observations we observed a dedicated field in the outer region of the globular cluster 47 Tuc to derive astrometric calibrations of IRDIS images (distortion, plate scale, and orientation), using the Hubble Space Telescope (HST) data as a reference (Bellini \& Anderson, priv. comm.; see Bellini et al. 2014 , for the methods used to obtain HST measurements). A detailed description of the astrometric calibration is presented in Maire et al. (2016).

The 47 Tuc data were reduced and analyzed using the Data Reduction and Handling software (DRH; Pavlov et al. 2008)
Table 3. Values of the plate scale and the true north for IRDIS observations measured during in each run of the observations.

\begin{tabular}{lcc}
\hline \hline Date & Plate scale (mas/px) & True north $(\mathrm{deg})$ \\
\hline \multicolumn{3}{c}{ Left field } \\
\hline July 2014 & $12.252 \pm 0.006$ & $-1.636 \pm 0.013$ \\
August 2014 & $12.263 \pm 0.006$ & $-1.636 \pm 0.013$ \\
December 2014 & $12.251 \pm 0.005$ & $-1.764 \pm 0.010$ \\
\hline \multicolumn{3}{c}{ Right field } \\
\hline July 2014 & $12.247 \pm 0.006$ & $-1.653 \pm 0.005$ \\
August 2014 & $12.258 \pm 0.006$ & $-1.653 \pm 0.005$ \\
December 2014 & $12.241 \pm 0.004$ & $-1.778 \pm 0.010$ \\
\hline
\end{tabular}

Notes. Values of July and August 2014 have been measured for the $H 2 H 3$ filter, while December values refer to BB_H band filter. The measurements for the left and right parts of the IRDIS detector are shown.

and IDL routines. To calculate the position of each star in the FoV, we used the IDL function cntrd $^{1}$ from the DAOphot software (Stetson 1987). We derived our IRDIS calibrations from the comparison between the measured positions on the detector of several tenths of stars and the HST observations (March 13, 2006). The typical accuracy of the catalog positions is $\sim 0.3$ mas and we took into account the time baseline between the HST and SPHERE observations. The distortion measured on sky is dominated by an anamorphism factor of $(0.60 \pm 0.02) \%$ between the horizontal and vertical directions of the detector. The anamorphism is common to both instruments because it is produced by cylindrical mirrors in the common path and infrastructure of the instrument.

Concerning IFS, as we did not obtain observations of any relevant astrometric calibrator, we calculated plate scale and orientation with a simultaneous observation of a distortion grid on both IRDIS and IFS detectors. We estimated for the IFS data a plate scale of $7.46 \pm 0.01 \mathrm{mas} / \mathrm{pix}$ and a relative orientation to IRDIS data of $-100.46 \pm 0.13 \mathrm{deg}$.

The astrometric calibrator sequences of 47 Tuc were acquired during the same observing runs as the science observations reported in this paper and with the same instrument setup (filter, coronagraph). Values calculated for the plate scale and for true north are shown in Table 3.

Raw data of both IRDIS and IFS have been corrected for anamorphism before the post-processing, taking into account that the IFS detector is rotated with respect to the IRDIS one.

The pixel scale variations from one observing run to another can be ascribed to ambient temperature variations. If we consider astrometric calibrator observations from the commissioning runs, we found the following relation between the

http://idlastro.gsfc.nasa.gov/ftp/pro/idlphot/ 


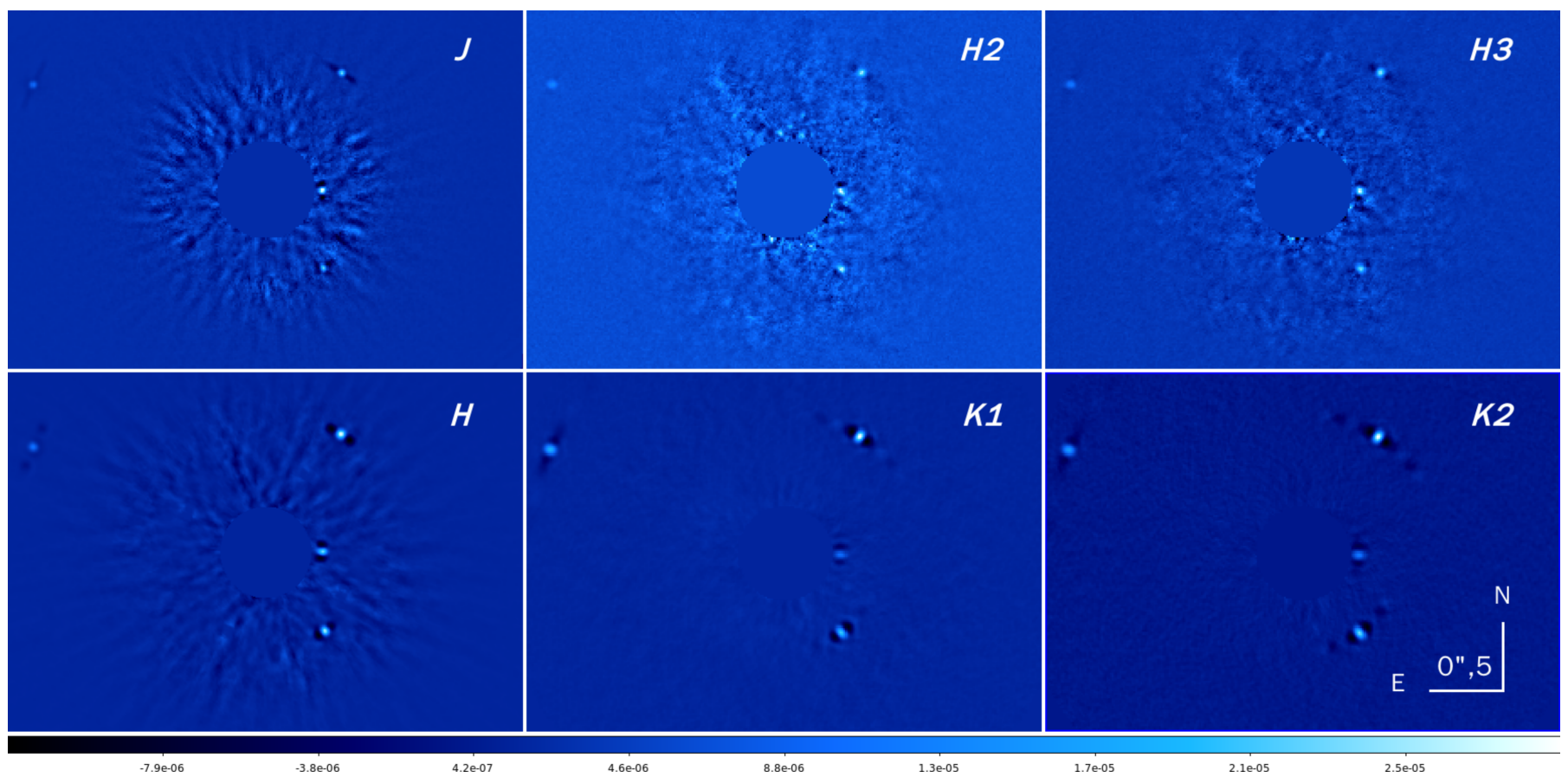

Fig. 1. IRDIS images for several filters with the four planets around HR 8799. The color scale is the same for all images, in unit of contrast with respect to the host star.

pixel scale and the temperature: scale $(\mathrm{mas} / \mathrm{px})=0.0060$ $T_{\mathrm{amb}}\left({ }^{\circ} \mathrm{C}\right)+12.1655$. The residual scatter is $0.012 \mathrm{mas} / \mathrm{px}$. This relation has been calculated with observations taken with the $H 2$ filter of IRDIS. For July data, the ambient temperature during the observations was on average $T_{\mathrm{amb}}=14.9^{\circ} \mathrm{C}$, which means that the expected pixel scale is $12.255 \pm 0.012$, compatible with the value listed in Table 3. We use this corrected value of the plate scale, which accounts for the temperature during the observations. The pixel scale of the filter $H 3$ is $12.250 \pm 0.012$.

\subsection{Broad and dual-band imaging}

The IRDIS data were preprocessed in the following way: first we subtracted from each datacube the corresponding sky background taken right after the sequence, we divided by flat-field images, and we applied a procedure to identify and interpolate bad-pixels. The frames were recentered using the initial star center exposure with the four satellite spots. The master backgrounds and master flat-field have been obtained with DRH software.

The second step of the reduction has been carried out with four independent pipelines performing ADI. We did not employ spectral differential imaging techniques (SDI; Racine et al. 1999) between the IRDIS filter pairs to avoid a flux loss that is not straightforward because of self-subtraction (Maire et al. 2014). The first data reduction method is based on the principal component analysis (PCA) technique that performs the Karhunen-Loève Image Projection (KLIP) algorithm, following the model of Soummer et al. (2012). This pipeline has been presented in Zurlo et al. (2014) and Vigan et al. (2016). For each product of the preprocessing phase, we created a PCA library based on all the other collapsed datacubes of the sequence, and we derotated the product of the PCA as a function of the parallactic angle of the reference datacube. A final image was obtained as the median of all the rotated PCA images. In some cases, the data was binned temporally to reduce the number of frames and increase the analysis speed.
The second pipeline is a more recent version of the T-LOCI algorithm presented in Marois et al. (2014). For each dual-band filter sequence, we calibrate the speckle pattern of each individual frame, rotate the frames to align north up, and median combined all the frames to obtain the final image.

To perform the reduction of the images in $J$ band, we exploited a third pipeline based on the method of the radial ADI (rADI) as illustrated in Chauvin et al. (2012).

Finally, the BB_H data were reduced with a fourth independent pipeline: datacubes were high-pass filtered by subtracting from each image a version of the image smoothed by a median filter with a large 11-pixel-wide box width before entering a PCA algorithm.

The final images obtained for all the IRDIS filters are shown in Fig. 1. No significant detection of a candidate planet is found closer than planet e in any of the images. The signal-to-noise ratio $(\mathrm{S} / \mathrm{N})$ for the planets goes from a minimum of 11 (planet $\mathrm{d}$ in $J$ band) to a maximum of 220 (planet c in $K 1$ band). Section 6.1 of Zurlo et al. (2014) gives a detailed explanation of how we calculated the $\mathrm{S} / \mathrm{N}$.

To measure photometry and astrometry, we exploited the method of the "fake negative planets" (Lagrange et al. 2010; Marois et al. 2010a; Bonnefoy et al. 2011), as illustrated in Zurlo et al. (2014) and Galicher et al. (2011). To build models of the planet images accounting for the ADI self-subtraction, we used the median of the unsaturated images of HR 8799 taken right before and after the sequences and the previously determined LOCI coefficients or PCA eigenvectors. No unsaturated images of HR 8799 were recorded during science verification, so HR 8799 b served as a PSF to build the planetary system model. We then adjusted the models to the real planet images to estimate the astrometry and photometry of the planets. We obtained the final results for each independent pipeline with a minimum $\chi^{2}$ fitting, where we simultaneously varied the flux and position of the fake negative planets (as described in Zurlo et al. 2014). The error bars for each reduction account for the flux variations of the unsaturated images of HR 8799, the fluctuations of the results as 
Table 4. IRDIS contrast in magnitudes and absolute magnitudes for HR 8799 bcde.

\begin{tabular}{lcccc}
\hline \hline Filter & $\mathrm{b}$ & $\mathrm{c}$ & $\mathrm{d}$ & $\mathrm{e}$ \\
\hline \multicolumn{5}{c}{ Contrast [mag] } \\
\hline$\Delta J$ & $14.39 \pm 0.09$ & $13.21 \pm 0.13$ & $13.20 \pm 0.37$ & $13.01 \pm 0.21$ \\
$\Delta H 2$ & $12.80 \pm 0.14$ & $11.81 \pm 0.12$ & $11.74 \pm 0.17$ & $11.63 \pm 0.20$ \\
$\Delta H 3$ & $12.50 \pm 0.10$ & $11.50 \pm 0.10$ & $11.57 \pm 0.16$ & $11.40 \pm 0.21$ \\
$\Delta K 1$ & $11.91 \pm 0.06$ & $10.95 \pm 0.05$ & $10.96 \pm 0.07$ & $10.88 \pm 0.10$ \\
$\Delta K 2$ & $11.73 \pm 0.09$ & $10.62 \pm 0.07$ & $10.60 \pm 0.10$ & $10.58 \pm 0.11$ \\
\hline \multicolumn{5}{c}{ Absolute magnitude [mag] } \\
\hline$J$ & $16.80 \pm 0.09$ & $15.62 \pm 0.13$ & $15.61 \pm 0.37$ & $15.42 \pm 0.21$ \\
$H 2$ & $15.10 \pm 0.14$ & $14.11 \pm 0.12$ & $14.04 \pm 0.17$ & $13.93 \pm 0.20$ \\
$H 3$ & $14.80 \pm 0.10$ & $13.80 \pm 0.10$ & $13.87 \pm 0.16$ & $13.70 \pm 0.21$ \\
$K 1$ & $14.17 \pm 0.06$ & $13.21 \pm 0.05$ & $13.22 \pm 0.07$ & $13.14 \pm 0.10$ \\
$K 2$ & $13.99 \pm 0.09$ & $12.88 \pm 0.07$ & $12.86 \pm 0.10$ & $12.84 \pm 0.11$ \\
\hline
\end{tabular}

Notes. The broad-band $H$ magnitudes are not provided as no off-axis PFS was taken during the sequence. This sequence is used for the astrometric analysis only in this paper. We refer to Apai et al., in prep. for a detailed discussion on the possible variability of these planets.

Table 5. Astrometric positions of the planets around HR 8799 in date 2014.53, derived from IRDIS filters $H 2 H 3$, and measurements in date 2014.62, from IFS $Y H$ band.

\begin{tabular}{|c|c|c|c|}
\hline Epoch & Planet & $\triangle \mathrm{RA}\left({ }^{\prime \prime}\right)$ & $\Delta \operatorname{Dec}\left({ }^{\prime \prime}\right)$ \\
\hline \multirow{4}{*}{2014.53} & HR 8799 b & $1.570 \pm 0.006$ & $0.707 \pm 0.006$ \\
\hline & HR 8799 c & $-0.522 \pm 0.004$ & $0.791 \pm 0.004$ \\
\hline & HR $8799 d$ & $-0.390 \pm 0.005$ & $-0.530 \pm 0.006$ \\
\hline & HR 8799 e & $-0.386 \pm 0.009$ & $-0.008 \pm 0.009$ \\
\hline \multirow[t]{2}{*}{2014.62} & HR 8799d & $-0.391 \pm 0.004$ & $-0.529 \pm 0.004$ \\
\hline & HR $8799 \mathrm{e}$ & $-0.384 \pm 0.002$ & $-0.005 \pm 0.002$ \\
\hline \multirow[t]{4}{*}{2014.93} & HR 8799 b & $1.574 \pm 0.005$ & $0.703 \pm 0.005$ \\
\hline & HR 8799c & $-0.518 \pm 0.004$ & $0.797 \pm 0.004$ \\
\hline & HR $8799 d$ & $-0.402 \pm 0.004$ & $-0.523 \pm 0.004$ \\
\hline & HR $8799 \mathrm{e}$ & $-0.384 \pm 0.010$ & $0.014 \pm 0.010$ \\
\hline
\end{tabular}

Notes. The planet positions in broad $H$-band in date 2014.93 are also shown.

a function of the PCA/LOCI parameters, and the accuracy of the fitting of the planet image models to the real images.

Results from each pipeline have been combined together excluding single results, which deviate more than the standard deviation, $\sigma$, of the values themselves. The error bars have been calculated as the quadratic sum of each independent reduction error bar plus the standard deviation of the values themselves. The contrast in magnitude obtained for the four planets is listed in Table 4, while their astrometric positions are shown in Table 5. The error budget is listed in Table 6 .

\subsection{IFS data reduction and spectra extraction}

We obtained IFS data in both $Y J(0.95-1.35 \mu \mathrm{m}$, spectral resolution $R \sim 54)$ and $Y H(0.95-1.65 \mu \mathrm{m}, R \sim 33)$ modes (Claudi et al. 2008). We only present the $Y H$ data because of the poor quality of the $Y J$ data obtained in July 2014. The IFS raw data were preprocessed using the DRH software, up to the creation of the calibrated spectral datacubes.

The preprocessing consists of background subtraction and flat-field calibration. Following this, the locations of the spectral traces are determined using a calibration image where the
Table 6. Astrometric error budget for both IRDIS and IFS measurements.

\begin{tabular}{lccccc}
\hline \hline Epoch & Planet & $\sigma_{\mathrm{PS}}\left({ }^{\prime \prime}\right)$ & $\sigma_{\text {Dith }}\left({ }^{\prime \prime}\right)$ & $\sigma_{\mathrm{SC}}\left({ }^{\prime \prime}\right)$ & $\sigma_{\mathrm{PP}}\left({ }^{\prime \prime}\right)$ \\
\hline 2014.53 & $\mathrm{~b}$ & 0.002 & 0.001 & 0.001 & 0.005 \\
& $\mathrm{c}$ & 0.001 & 0.001 & 0.001 & 0.004 \\
& $\mathrm{~d}$ & 0.001 & 0.001 & 0.001 & 0.005 \\
& $\mathrm{e}$ & 0.000 & 0.001 & 0.001 & 0.009 \\
\hline \multirow{2}{2014.62}{} & $\mathrm{d}$ & 0.001 & - & 0.001 & 0.004 \\
& $\mathrm{e}$ & 0.001 & - & 0.001 & 0.001 \\
\hline 2014.93 & $\mathrm{~b}$ & 0.002 & 0.001 & 0.001 & 0.004 \\
& $\mathrm{c}$ & 0.001 & 0.001 & 0.001 & 0.004 \\
& $\mathrm{~d}$ & 0.001 & 0.001 & 0.001 & 0.004 \\
& $\mathrm{e}$ & 0.000 & 0.001 & 0.001 & 0.010 \\
\hline
\end{tabular}

Notes. For each planet the error was calculated as a combination of: plate scale uncertainty $\left(\sigma_{\mathrm{PS}}\right)$; dithering procedure accuracy $\left(\sigma_{\text {Dith }}=\right.$ 0.74 mas, here approximated to 1 mas); star center position uncertainty $\left(\sigma_{\mathrm{SC}}\right)$ of 1.2 mas, derived from observation of bright stars during commissioning runs; planet position uncertainty $\left(\sigma_{\mathrm{PP}}\right)$. For the sake of simplicity we listed just the error along the $x$ axis of the detector for the planet position measurement. The errors due to the anamorphism uncertainty and the true north uncertainty are less than 0.5 mas for any planet, so they are not listed here, but included in the calculation of the error budget.

integral field unit (IFU) is uniformly illuminated with a white lamp in such a way that the detector is completely filled with spectra. For wavelength calibration, the IFU is illuminated with four monochromatic lasers of known wavelength. An instrument flat is used to correct for the different response of the lenslets to a uniform illumination and detector quantum efficiency variations. After this last procedure, we are left with calibrated spectral datacubes comprised of 39 monochromatic images.

An additional step using custom IDL tools has been introduced for better handling of bad pixels and to implement spectral cross-talk corrections (Mesa et al. 2015). After the creation of the calibrated spectral datacubes, the differential imaging part of the data reduction is performed applying different pipelines written in IDL.

We have adopted two different approaches to searching for additional companions in the HR 8799 system as well as extracting spectra of the detected planets. Both approaches provided detection of planets $d$ and e with a median $\mathrm{S} / \mathrm{N}$ along the IFS channels of $\sim 20$. To search for additional planets, we utilize the KLIP algorithm, exploiting all the frames at different wavelengths and at different angular positions. No significant signal has been found closer than planet e.

For extraction of spectra of known objects, we first utilize ADI separately for each individual IFS channel. As shown in Table 2, for the first $Y H$ observations we took 16 datacubes comprised of three frames with a DIT of $60 \mathrm{~s}$, while for the second observations we took 16 datacubes comprised of two frames with a DIT of $100 \mathrm{~s}$. We decided to use all 80 frames without performing any binning in time, rescaling the frames of the second data set to the total integration time of the first. For each spectral frame we performed PCA fitting using the frames of the same channel of the other datacubes as a library. To avoid self-subtraction, we selected the frames according to the planet position in each image of the library, and rejected the frames where the planet centroid moved less than $\sim 0.5 \lambda / D$ with respect to the reference frame, following the example presented in Pueyo et al. (2015). For each planet the library is composed of a different set of frames, however, we also attempted data reductions 


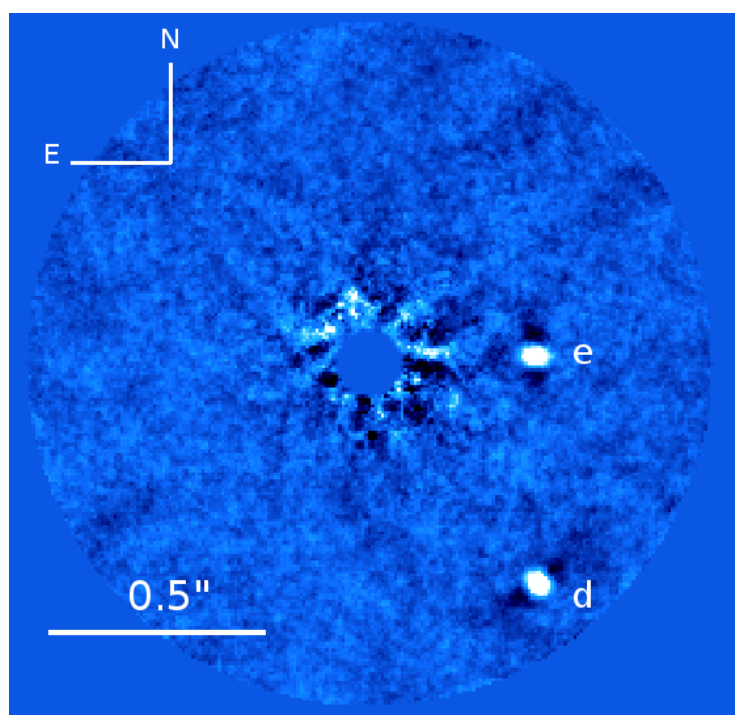

Fig. 2. 38th IFS spectral channel $(1.63 \mu \mathrm{m})$ with planets HR 8799 de obtained with KLIP reduction.

without performing any selection of the frames. In this case, we used a small number of modes to limit the biases of the PCA. We used different number of modes $(<80)$, but the result converged rapidly after a few modes (between 2 and 5). To retrieve the signal of a planet, we tested the impact of our reductions with synthetic planets injected in the preprocessed datacubes at different positions or in the forward modeling (see description in Soummer et al. 2012), assuming the same PCA modes and aperture as employed for the spectral extraction in the science data. For the forward modeling, we used the off-axis PSF obtained after the observing sequence because of a slight saturation of the off-axis PSF obtained before the observation. An example of the output of this technique is shown in Fig 2.

A second approach, where ADI and SDI were used together, was then implemented. The single exposures in each of the 32 spectral datacubes were averaged. The PSF library used for the PCA fitting taking all frames into account, both along the temporal and spectral dimensions, where the PSF has moved by more than a separation criteria $N_{\delta}$. In this scheme, the library is constructed using the frames spatially rescaled according to their respective wavelength. Various tests showed that a value of $N_{\delta}=0.8 \mathrm{FWHM}$ gives good results in terms of $\mathrm{S} / \mathrm{N}$ for the detection of the planets. To account for the flux losses induced by the speckle subtraction algorithm, forward modeling is performed via the off-axis PSF of the above-mentioned star as a model for the PSF of the planet. The model is projected on the same PCA modes as the science data, and the flux loss is measured as the attenuation of the flux for the model before and after projection on the PCA modes. The fluxes of the planets are measured in the final spectral channels with aperture photometry in an aperture of diameter $0.8 \lambda / D$ ( $\sim 4$ pixels). The flux is finally corrected for the flux loss estimated using the forward modeling. The error bars on the photometry were measured in the same way as for the IRDIS data described in Sect. 3.2, except that we did not consider the flux variation during the exposure, as one of the two PSFs is slightly saturated.

With this approach, we obtained eight spectral extractions from four independent pipelines. For each planet, a final estimate of the flux ratio between the star and planet was obtained by computing the median of the results.

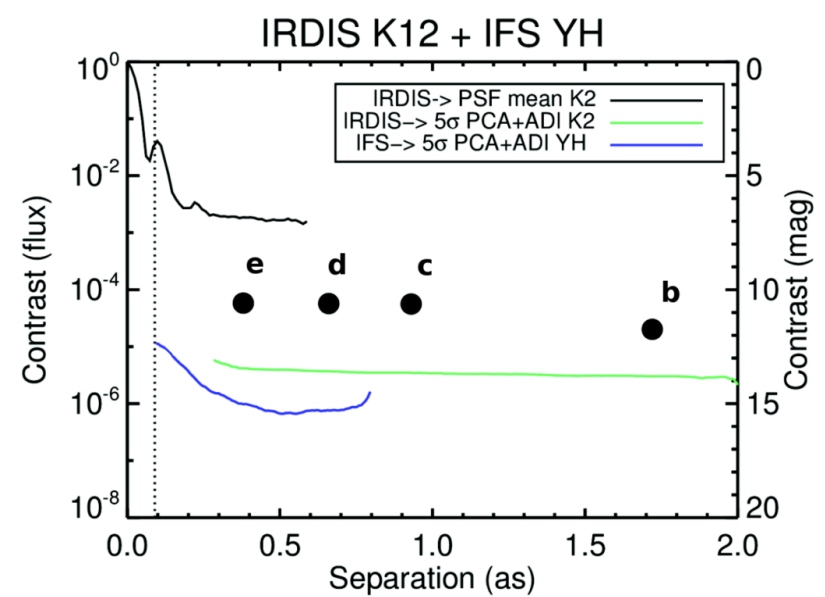

Fig. 3. Contrast and off-axis PSFs curves for the IRDIFS_EXT data set. The $5 \sigma$ contrast for the two instruments are plotted as a function of the angular separation. The off-axis PSF of the star is represented as a black continuous line. Planets around HR 8799 are also shown as photometric $K 2$ points, the error bars are inside the dimension of the dots. The dotted vertical line indicates the coronagraph IWA.

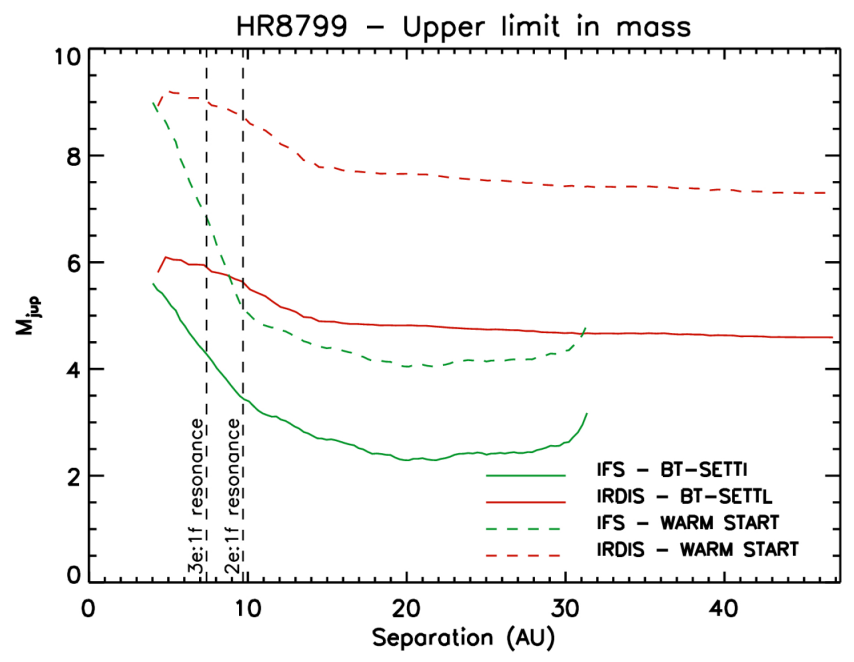

Fig. 4. Mass limits deduced from the contrast curves for the IRDIS and IFS instruments. An age of $30 \mathrm{Myr}$ is assumed. Two different models are represented: the "hot-start models" BT-SETTL models, and the "warm-start models" from Spiegel \& Burrows (2012). The separations where a possible planet $\mathrm{f}$ is expected to be (from Goździewski \& Migaszewski 2014) are shown as dashed black lines.

Astrometric measurements for planets HR 8799 de measured from the IFS are shown in Table 5. The error budget is presented in Table 6. The error bars are compatible with the expected ones for these targets, following the simulations of Zurlo et al. (2014).

The $5 \sigma$ contrast curves of IRDIS and IFS for the IRDIFS_EXT data set are shown in Fig. 3. The limits were calculated by measuring the standard deviation of the residuals in annuli of width $1 \lambda / \mathrm{D}$ at increasing angular separation, normalized for the flux of the corresponding PSF.

Contrast in the $K 2$ band of the planets around HR 8799 are overplotted. IRDIS contrast curve has been calculated on the KLIP product of the filter $K 2$, while the IFS contrast curve has been obtained on the KLIP result with just ADI ( $Y H$ band).

The mass limits deduced from the contrast curves of Fig. 3 are shown in Fig. 4. To calculate the mass limits, we assumed an age of $30 \mathrm{Myr}$ for the system and two different models: the 
BT-SETTL (Allard et al. 2012) for "hot-start models" as well as the "warm-start models" from Spiegel \& Burrows (2012).

Following Goździewski \& Migaszewski (2014), a possible planet could be located at the 3e:1f and 2e:1f resonance orbits, corresponding to separation of $\sim 7.5$ and $\sim 10$ AU. The two separations are overplotted in Fig. 4. In their analysis they found that a planet of mass 2-8 $M_{\text {Jup }}$ in the $3 \mathrm{e}: 1 \mathrm{f}$ orbit and $1.5-5 M_{\mathrm{Jup}}$ at the 2e:1f orbit could be present without perturbations on the stability of the system. We found that with IFS we are able to detect planets with mass of 4.5-7 $M_{\text {Jup }}$ at the separation of the 3e:1f resonance and 3-5 $M_{\text {Jup }}$ at the resonance $2 \mathrm{e}: 1 \mathrm{f}$. This means that 2-4.5 $M_{\text {Jup }}$ planets are not excluded by our estimations for the $3 \mathrm{e}: 1 \mathrm{f}$ orbit, as we do not exclude $1.5-3 M_{\text {Jup }}$ planets for the 2e:1f orbit.

\section{Astrometric fit of the orbits}

In this section, we present an illustration of the accuracy of SPHERE in measuring the astrometric positions of low-mass companions with respect to the previous generation of highcontrast imagers. We performed an astrometric fit for the orbits of planets HR 8799 bcde. The aim of this section is to present sample orbital solutions compatible with the astrometric data, but a detailed orbital analysis is beyond the scope of this paper.

We combined the astrometric positions available from the literature, including Marois et al. (2008, 2010b), Lafrenière et al. (2009), Fukagawa et al. (2009), Hinz et al. (2010), Currie et al. (2011), Bergfors et al. (2011), Galicher et al. (2011), Soummer et al. (2011), Esposito et al. (2013), Currie et al. (2012, 2014), Maire et al. (2015), Pueyo et al. (2015) with our SPHERE data (Table 5). We rely on the nominal error bars from the individual references, although systematic errors between different instruments and analysis procedures may be present. The SPHERE astrometric measurements are compatible with the literature data and achieve an accuracy similar or better with respect to the published measurements, which is a good test for this new instrument.

The orbital fit is a minimum $\chi^{2}$ fitting, as described in Esposito et al. (2013), where all the four orbits are simultaneously taken into consideration, using the assumption that the planet periods are commensurate. The IFS measurement is used for the closest planet e, as it provides smaller error bars. We assumed circular and non-coplanar orbits and tested three different configurations for the orbital period ratios between the planets.

The fitted orbits are shown in Fig. 5. The parameters of the orbital solutions are summarized in Table 7. The uncertainties on the parameters were estimated using a quadratic combination of statistical and systematic errors. The statistical errors stem from the sensitivity of the fit to the errors bars on the astrometric measurements and are derived from 1000 random trials of the astrometric measurements assuming normal distributions (Maire et al. 2015). The systematic errors are related to the uncertainties on the stellar mass and distance (Table 7), which propagate into the derivation of the semimajor axis. To assess them, we performed two sets of orbital fits. The first set assumes the nominal values for the stellar mass and distance, and the second set assumes a combination of values at the edge of the derived ranges, which provides the largest offset in orbital periods. Two orbital solutions are favored with similar reduced $\chi^{2}$, with the orbital period ratios $2 \mathrm{~d}: 1 \mathrm{e}$ and $3 \mathrm{~d}: 1 \mathrm{e}$. The IFS data point for planet e is out of the orbital solution with the orbital period ratio $5 \mathrm{~d}: 2 \mathrm{e}$ at $\sim 2.5 \sigma$ significance level. Further astrometric monitoring is required to distinguish between the orbital period ratios $2 \mathrm{~d}: 1 \mathrm{e}$ and $3 \mathrm{~d}: 1 \mathrm{e}$.
Table 7. Orbital elements fitted on the astrometric data in Fig. 5.

\begin{tabular}{|c|c|c|c|}
\hline Parameter & A & B & $\mathrm{C}$ \\
\hline \multicolumn{4}{|l|}{ HR 8799 b } \\
\hline$P(\mathrm{yr})$ & $455.88 \pm 24.70$ & $455.88 \pm 24.70$ & $455.88 \pm 24.70$ \\
\hline$i\left(^{\circ}\right)$ & $30.27 \pm 4.95$ & $30.27 \pm 4.95$ & $30.27 \pm 4.95$ \\
\hline$\Omega\left({ }^{\circ}\right)$ & $60.89 \pm 4.40$ & $60.89 \pm 4.40$ & $60.89 \pm 4.40$ \\
\hline$e$ & - & - & - \\
\hline$\omega\left(^{\circ}\right)$ & - & - & - \\
\hline$T 0(\mathrm{yr})$ & $2006.55 \pm 6.44$ & $2006.55 \pm 6.44$ & $2006.55 \pm 6.44$ \\
\hline$a(\mathrm{AU})$ & $67.96 \pm 1.85$ & $67.96 \pm 1.85$ & $67.96 \pm 1.85$ \\
\hline \multicolumn{4}{|l|}{ HR 8799 c } \\
\hline$P(\mathrm{yr})$ & $227.94 \pm 12.35$ & $227.94 \pm 12.35$ & $227.94 \pm 12.35$ \\
\hline$i\left(^{\circ}\right)$ & $29.43 \pm 0.35$ & $29.43 \pm 0.35$ & $29.43 \pm 0.35$ \\
\hline$\Omega\left({ }^{\circ}\right)$ & $65.68 \pm 2.67$ & $65.68 \pm 2.67$ & $65.68 \pm 2.67$ \\
\hline$e$ & - & - & - \\
\hline$\omega\left(^{\circ}\right)$ & - & - & - \\
\hline$T 0(\mathrm{yr})$ & $1848.18 \pm 9.84$ & $1848.18 \pm 9.84$ & $1848.18 \pm 9.84$ \\
\hline$a(\mathrm{AU})$ & $42.81 \pm 1.16$ & $42.81 \pm 1.16$ & $42.81 \pm 1.16$ \\
\hline \multicolumn{4}{|l|}{ HR $8799 \mathrm{~d}$} \\
\hline$P(\mathrm{yr})$ & $113.97 \pm 6.18$ & $113.97 \pm 6.18$ & $113.97 \pm 6.18$ \\
\hline$i\left(^{\circ}\right)$ & $38.63 \pm 2.84$ & $38.63 \pm 2.84$ & $38.63 \pm 2.84$ \\
\hline$\Omega\left({ }^{\circ}\right)$ & $56.09 \pm 3.78$ & $56.09 \pm 3.78$ & $56.09 \pm 3.78$ \\
\hline$e$ & - & - & - \\
\hline$\omega\left(^{\circ}\right)$ & - & - & - \\
\hline$T 0(\mathrm{yr})$ & $1965.27 \pm 1.55$ & $1965.27 \pm 1.55$ & $1965.27 \pm 1.55$ \\
\hline$a(\mathrm{AU})$ & $26.97 \pm 0.73$ & $26.97 \pm 0.73$ & $26.97 \pm 0.73$ \\
\hline \multicolumn{4}{|l|}{ HR 8799 e } \\
\hline$P(\mathrm{yr})$ & $56.99 \pm 3.09$ & $46.99 \pm 2.79$ & $75.19 \pm 4.05$ \\
\hline$i\left(^{\circ}\right)$ & $30.95 \pm 1.43$ & $28.43 \pm 5.39$ & $44.51 \pm 0.45$ \\
\hline$\Omega\left({ }^{\circ}\right)$ & $145.73 \pm 7.11$ & $85.50 \pm 3.47$ & $156.08 \pm 2.54$ \\
\hline$e$ & - & - & - \\
\hline$\omega\left(^{\circ}\right)$ & - & - & - \\
\hline$T 0(\mathrm{yr})$ & $1995.61 \pm 0.93$ & $1990.54 \pm 1.02$ & $1992.31 \pm 1.17$ \\
\hline$a(\mathrm{AU})$ & $16.99 \pm 0.46$ & $14.94 \pm 0.46$ & $20.44 \pm 0.55$ \\
\hline$\chi_{\text {red,b }}^{2}$ & 2.33 & 2.33 & 2.33 \\
\hline$\chi_{\text {red,c }}^{2}$ & 2.04 & 2.04 & 2.04 \\
\hline$\chi_{\text {red,d }}^{2}$ & 1.92 & 1.92 & 1.92 \\
\hline$\chi_{\text {red,e }}^{2}$ & 1.21 & 3.44 & 1.18 \\
\hline$M_{\text {star }}\left(M_{\odot}\right)$ & $1.51_{-0.024}^{+0.038}$ & $1.51_{-0.024}^{+0.038}$ & $1.51_{-0.024}^{+0.038}$ \\
\hline$d(\mathrm{pc})$ & $39.4 \pm 1.1$ & $39.4 \pm 1.1$ & $39.4 \pm 1.1$ \\
\hline
\end{tabular}

Notes. Case A: orbital solution setting circular orbits and $8 \mathrm{~b}: 4 \mathrm{c}: 2 \mathrm{~d}: 1 \mathrm{e}$ mean motion resonance. Case B: orbital solution setting circular orbits and $4 \mathrm{~b}: 2 \mathrm{c}: 1 \mathrm{~d}$ and $5 \mathrm{~d}: 2 \mathrm{e}$ mean motion resonances. Case C: orbital solution setting circular orbits and $4 \mathrm{~b}: 2 \mathrm{c}: 1 \mathrm{~d}$ and $3 \mathrm{~d}: 2 \mathrm{e}$ mean motion resonances. For each planet, the notations refer to the orbital period, inclination, longitude of the ascending node, eccentricity, argument of periapsis, time of periapsis passage, and semimajor axis. The error bars on the orbital parameters are at $1 \sigma$ (see text). We also indicate the reduced $\chi^{2}$ for the nominal orbital solution for each planet, and the mass and distance ranges assumed for the host star for the orbital fitting (Baines et al. 2012; van Leeuwen 2007). The semimajor axes are derived using Kepler's third law assuming the fitted orbital periods and that the planet masses are negligible with respect to the mass of the host star.

\section{Comparison to known objects}

In this section, we used the extracted photometry from IRSDIS and spectrophotometry from the IFS to explore characterization of the spectral energy distributions (SEDs) of the planetary mass 

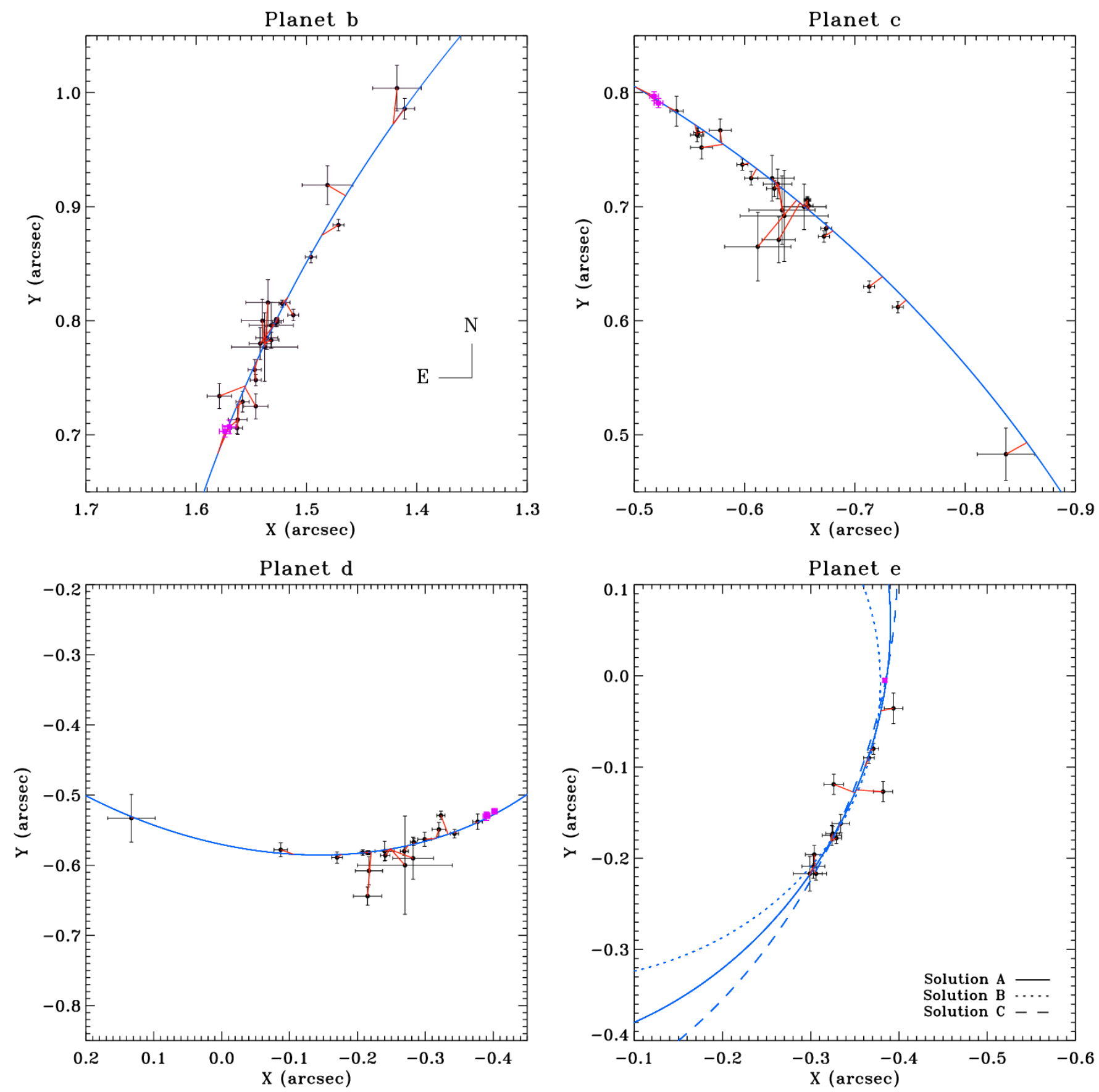

Fig. 5. Relative astrometry for the planets around HR 8799. The points available from the literature are represented with black filled circles, while new SPHERE measurements are represented with purple filled squares. The solid blue lines represent the orbital solution labeled A in Table 7. Red lines connect the predicted and observed positions for all the data points. The dotted and dashed lines in the panel for HR 8799 e indicate the orbital solutions labeled B and C in Table 5, respectively.

objects surrounding HR 8799. An in-depth study of these properties is presented in a companion paper (Bonnefoy et al. 2016).

\subsection{Colors}

We first compare the IRDIS colors of HR 8799 bcde to those of MLT field dwarfs. The colors of the field dwarfs were synthetized from SpeXPrism spectral library ${ }^{2}$. For that purpose, we smoothed the low-resolution spectra of the sources to the

\footnotetext{
2 http://pono.ucsd.edu/ adam/browndwarfs/spexprism/
}

resolution of the narrowest IRDIS filter, using the IRDIS filter passbands, a model of the Paranal atmospheric transmission generated with the ESO Skycalc web application ${ }^{3}$ (Noll et al. 2012; Jones et al. 2013), and a model spectrum of Vega (Bohlin 2007).

Certain two-color diagrams enable discrimination of field dwarf objects with different spectral types at the L-T transition. They are shown in Figs. 6 and 7. The planets are located at the transition between late-L and early- $\mathrm{T}$ dwarfs, but have redder colors than the dwarf objects (i.e. are brighter in the $K$-band than

\footnotetext{
3 http://www . eso.org/observing/etc/bin/gen/form?INS. MODE=swspectr+INS . NAME=SKYCALC
} 
A. Zurlo et al.: SPHERE results on the HR 8799 planetary system

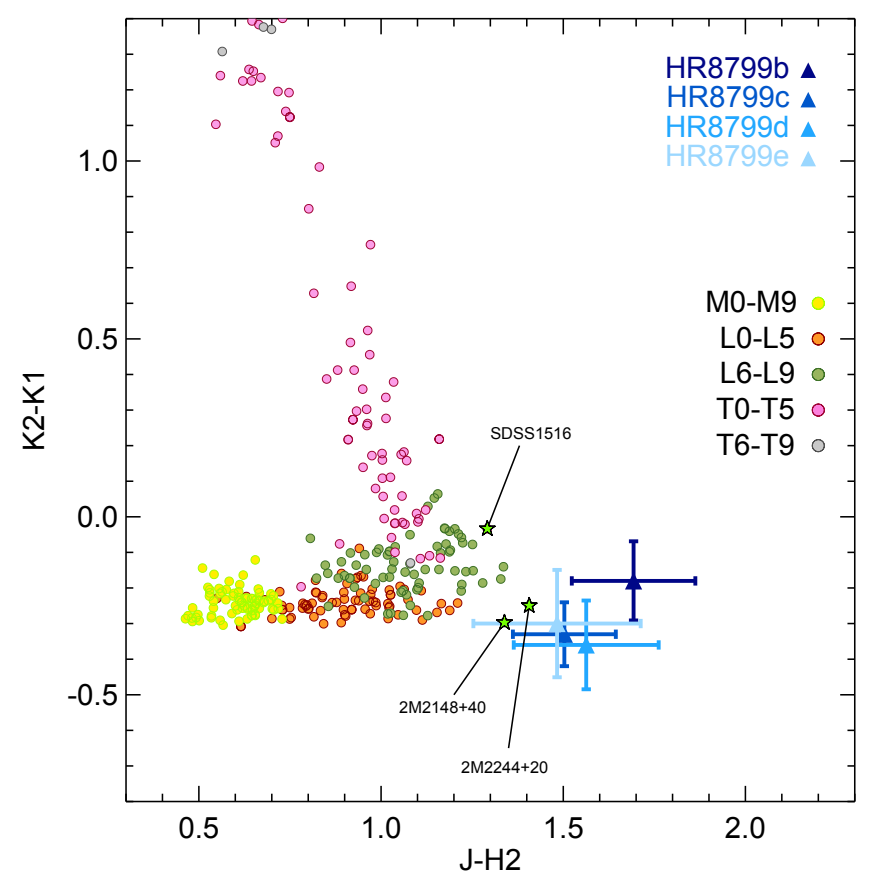

Fig. 6. Comparison of HR 8799 bcde colors based on IRDIS photometry to those of M, L, T field dwarfs (dots).

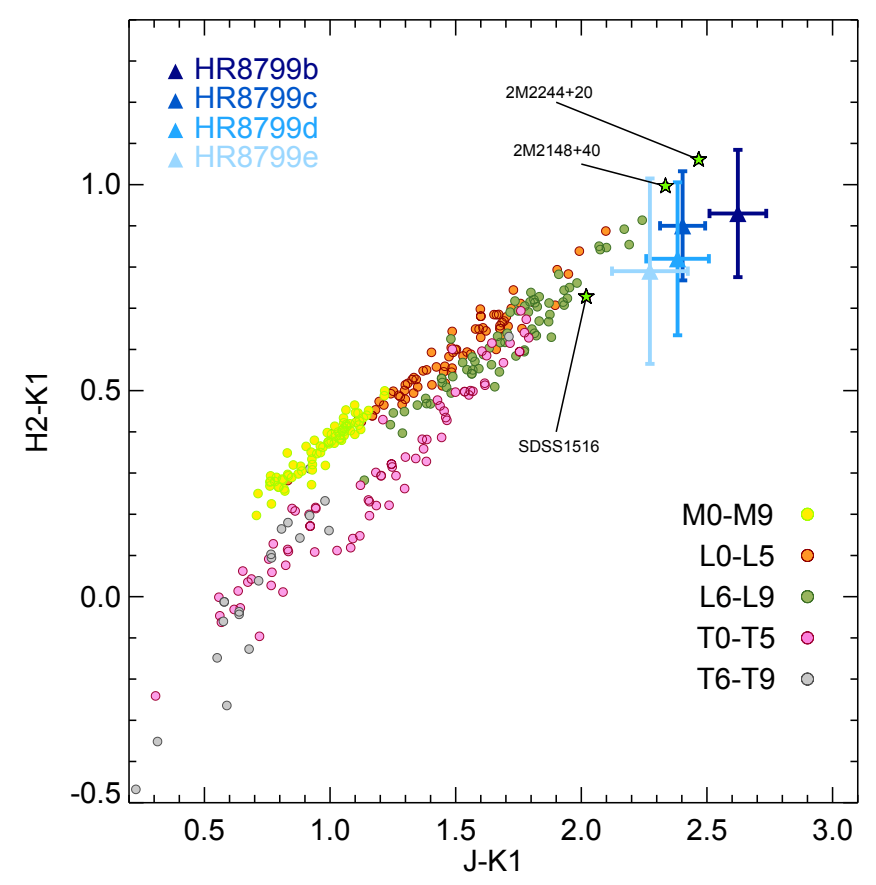

Fig. 7. Same as Fig. 6, except for the $J-K 1$ versus $H 2-K 1$ colors.

expected). This is in agreement with the location of the objects in color-magnitude diagrams (Marois et al. 2008; Currie et al. 2011).

\subsection{Near-infrared slope}

We converted the flux-ratio (IFS spectra, IRDIS photometry) between the HR 8799 planets and their host star into apparent flux, corrected from telluric absorptions with a model spectrum of the star (ATLAS9 Model grid; Castelli \& Kurucz 2004) with

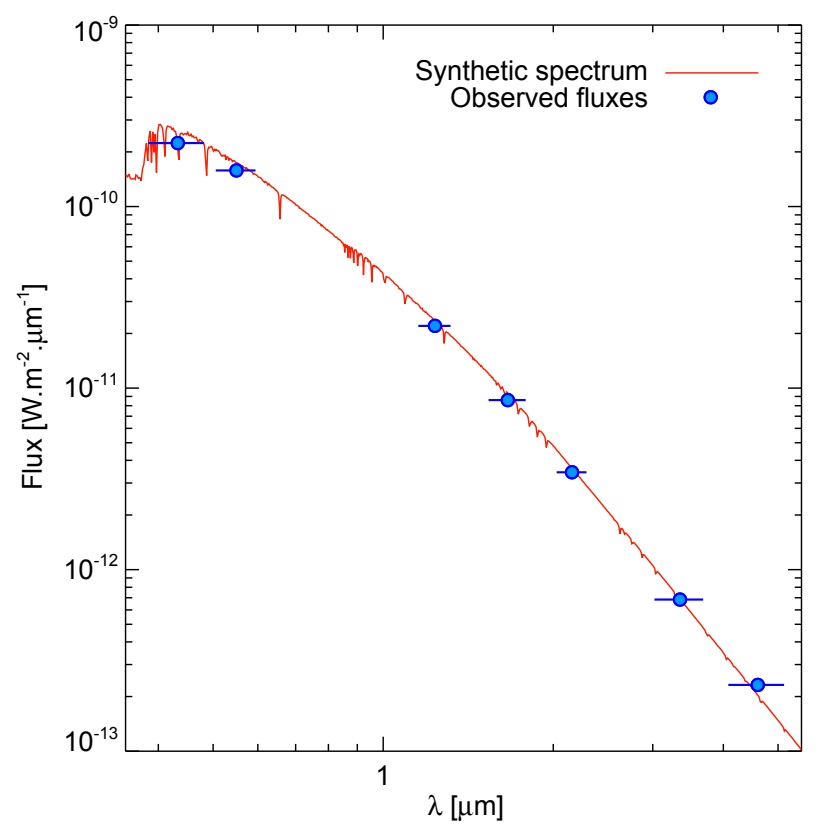

Fig. 8. Synthetic ATLAS9 spectrum adjusted to the apparent fluxes of HR 8799 A.

$T_{\text {eff }}=7500 \mathrm{~K}, \log g=4.5, M / H=0, v_{\text {turb }}=0 \mathrm{~km} \mathrm{~s}^{-1}$ scaled in flux to fit the Tycho $2 \mathrm{BV}, 2 \mathrm{MASS} J H K_{\mathrm{S}}$, and WISE $W 1 W 2$ photometry (Høg et al. 2000; Cutri et al. 2003, 2012). This synthetic spectrum was found to provide a good fit for the existing photometry. The scaled spectrum of the star is shown in Fig. 8.

We used this flux-calibrated model spectrum of HR 8799 A to retrieve the flux of HR 8799 bcde in the IRDIS passbands (Table 8) and to obtain flux-calibrated spectra of the two innermost planets. Figures 9 and 10 show that the $J, H 2$, and $H 3$ photometry of HR $8799 \mathrm{~d}$ and e is consistent with the spectra extracted from the IFS datacubes. We also included the flux-calibrated $K$-band GPI spectrum of HR 8799 d obtained by Ingraham et al. (2014). This spectrum is compatible with IRDIS $K 1$ and $K 2$ photometry of the planet.

The spectra of the two planets together are shown in Fig. 11.

A comparison of the two spectra with results from Project 1640 (Oppenheimer et al. 2013) is shown in Fig. 12. A table with the flux values for each IFS channel is available in the electronic version of this paper.

We compared the flux-calibrated spectra, $H 2, H 3, K 1$, and $K 2$ fluxes of HR $8799 \mathrm{~d}$ and e to those of field brown dwarfs from Testi et al. (2001) and to 1000 empirical templates from the SpeXPrism library. While the Testi et al. (2001) library mostly contains spectra of old objects, the SpeXPrism library includes spectra of some dusty dwarf and peculiar (red) L and T dwarfs. The comparison illustrates, as previously found in the literature, that the planet properties are best reproduced by objects at the $\mathrm{L} / \mathrm{T}$ transition. The spectral shapes of the SPHERE/IFS spectra of the two innermost planets are reproduced by those of L6 field dwarfs (Figs. 9 and 10). Nevertheless, the planet spectra have higher fluxes than these field dwarfs at longer wavelengths (i.e. their spectral slopes are redder).

Among the sample of SpeXPrism spectra, those of 2MASS J22443167+2043433 (L7.5/L6.5, Dahn et al. 2002) and SDSS J151643.01+305344.4 (T0.5-1.5pec, Chiu et al. 2006; Burgasser et al. 2010) best match the spectra of HR8799d and e, respectively. Barman et al. (2011) has already noted the similarity of SDSS $1516+30$ to HR 8799 b. Both SDSS $1516+30$ 
Table 8. Currently available fluxes of HR $8799 \mathrm{~b}$ at $10 \mathrm{pc}$ gathered from narrowband and broadband photometry.

\begin{tabular}{cccccc}
\hline \hline Filter & $\begin{array}{c}\lambda \\
(\mu \mathrm{m})\end{array}$ & $\begin{array}{c}F W H M \\
(\mu \mathrm{m})\end{array}$ & $\begin{array}{c}\text { Abs. flux } \\
\left(\mathrm{Wm}^{-2} \mu \mathrm{m}^{-1}\right)\end{array}$ & $\begin{array}{c}\text { Err. flux }+ \\
\left(\mathrm{Wm}^{-2} \mu \mathrm{m}^{-1}\right)\end{array}$ & $\begin{array}{c}\text { Err. flux }- \\
\left(\mathrm{Wm}^{-2} \mu \mathrm{m}^{-1}\right)\end{array}$ \\
\hline BB_J & 1.25 & 0.20 & $6.42 \mathrm{E}-16$ & $6.72 \mathrm{E}-17$ & $6.09 \mathrm{E}-17$ \\
$H 2$ & 1.59 & 0.05 & $1.26 \mathrm{E}-15$ & $1.90 \mathrm{E}-16$ & $1.65 \mathrm{E}-16$ \\
$H 3$ & 1.67 & 0.06 & $1.42 \mathrm{E}-15$ & $1.61 \mathrm{E}-16$ & $1.44 \mathrm{E}-16$ \\
$K 1$ & 2.11 & 0.10 & $1.05 \mathrm{E}-15$ & $8.54 \mathrm{E}-17$ & $7.90 \mathrm{E}-17$ \\
$K 2$ & 2.25 & 0.11 & $9.71 \mathrm{E}-16$ & $1.02 \mathrm{E}-16$ & $9.21 \mathrm{E}-17$ \\
\hline & & & HR 8799c & \\
\hline BB_J & 1.25 & 0.20 & $1.90 \mathrm{E}-15$ & $2.68 \mathrm{E}-16$ & $2.35 \mathrm{E}-16$ \\
$H 2$ & 1.59 & 0.05 & $3.15 \mathrm{E}-15$ & $4.14 \mathrm{E}-16$ & $3.66 \mathrm{E}-16$ \\
$H 3$ & 1.67 & 0.06 & $3.56 \mathrm{E}-15$ & $4.03 \mathrm{E}-16$ & $3.62 \mathrm{E}-16$ \\
$K 1$ & 2.11 & 0.10 & $2.54 \mathrm{E}-15$ & $1.90 \mathrm{E}-16$ & $1.76 \mathrm{E}-16$ \\
$K 2$ & 2.25 & 0.11 & $2.70 \mathrm{E}-15$ & $2.39 \mathrm{E}-16$ & $2.20 \mathrm{E}-16$ \\
\hline & & & HR 8799d & \\
\hline BB_J & 1.25 & 0.20 & $1.92 \mathrm{E}-15$ & $7.92 \mathrm{E}-16$ & $5.61 \mathrm{E}-16$ \\
$H 2$ & 1.59 & 0.05 & $3.35 \mathrm{E}-15$ & $6.06 \mathrm{E}-16$ & $5.13 \mathrm{E}-16$ \\
$H 3$ & 1.67 & 0.06 & $3.34 \mathrm{E}-15$ & $5.69 \mathrm{E}-16$ & $4.86 \mathrm{E}-16$ \\
$K 1$ & 2.11 & 0.10 & $2.52 \mathrm{E}-15$ & $2.23 \mathrm{E}-16$ & $2.05 \mathrm{E}-16$ \\
$K 2$ & 2.25 & 0.11 & $2.75 \mathrm{E}-15$ & $3.12 \mathrm{E}-16$ & $2.80 \mathrm{E}-16$ \\
\hline & & & & \\
\hline BB_J & 1.25 & 0.20 & $2.29 \mathrm{E}-15$ & $5.10 \mathrm{E}-16$ & $4.17 \mathrm{E}-16$ \\
$H 2$ & 1.59 & 0.05 & $3.71 \mathrm{E}-15$ & $7.87 \mathrm{E}-16$ & $6.50 \mathrm{E}-16$ \\
$H 3$ & 1.67 & 0.06 & $3.90 \mathrm{E}-15$ & $8.69 \mathrm{E}-16$ & $7.11 \mathrm{E}-16$ \\
$K 1$ & 2.11 & 0.10 & $2.71 \mathrm{E}-15$ & $3.07 \mathrm{E}-16$ & $2.76 \mathrm{E}-16$ \\
$K 2$ & 2.25 & 0.11 & $2.80 \mathrm{E}-15$ & $3.42 \mathrm{E}-16$ & $3.05 \mathrm{E}-16$ \\
\hline
\end{tabular}

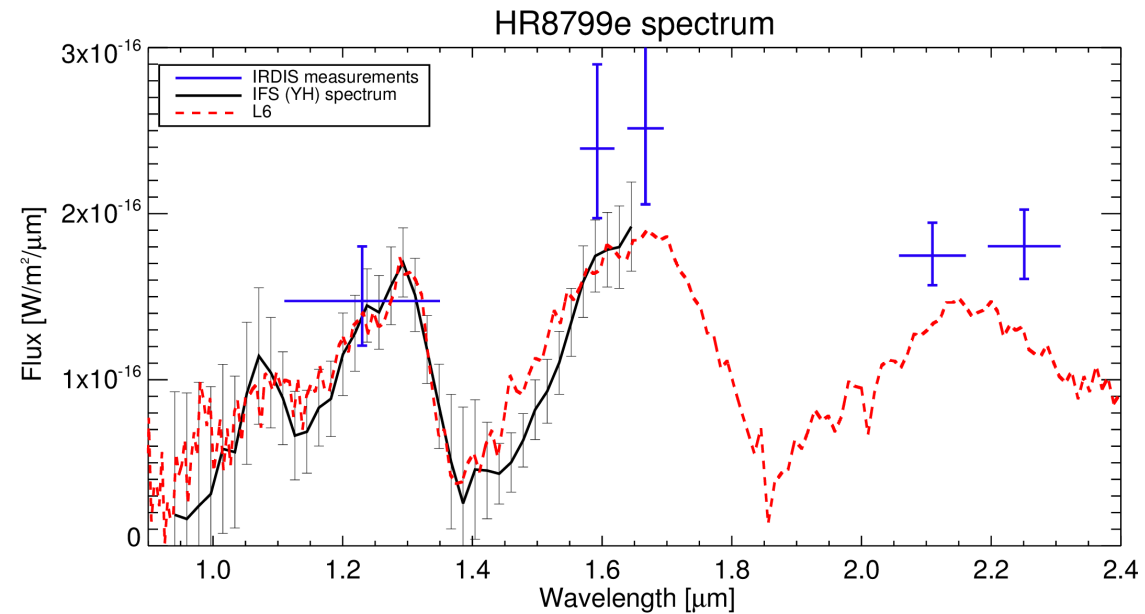

Fig. 9. Spectrum of planet e as extracted from IFS data. IRDIS photometric points are overplotted. A comparison with an L6 isolated brown dwarf shows a mismatch at $K$ band wavelengths. There is a slight mismatch between IRDIS and IFS measurements, as we separately normalized the flux of the two detectors with the corresponding PSF, the offset could be induced by another variable.

and 2MASS $2244+20$ are shown in Figs. 6 and 7. The colors of 2MASS J22443167+2043433, along with the peculiar L6 dwarf 2MASS J21481633+4003594 (Looper et al. 2008), deviate from the sequence of field dwarfs. These objects are red dusty dwarfs proposed to be young members of the field population (Stephens et al. 2009; Gagné et al. 2014, and references therein).

In summary, the analysis demonstrates that there is a deviation of HR 8799 bcde spectrophotometric properties from the sequence of field dwarfs. The properties of HR $8799 \mathrm{~d}$ and e are shared by some red dusty, and possibly young, objects at the $\mathrm{L} / \mathrm{T}$ transition. We investigate these properties in more detail in the companion paper, Bonnefoy et al. (2016).

\section{Conclusions}

We present first results of the planet hunter SPHERE on the planetary mass objects surrounding the young intermediate mass star HR 8799. We observed this system during the commissioning and the science verification runs of SPHERE during the months 


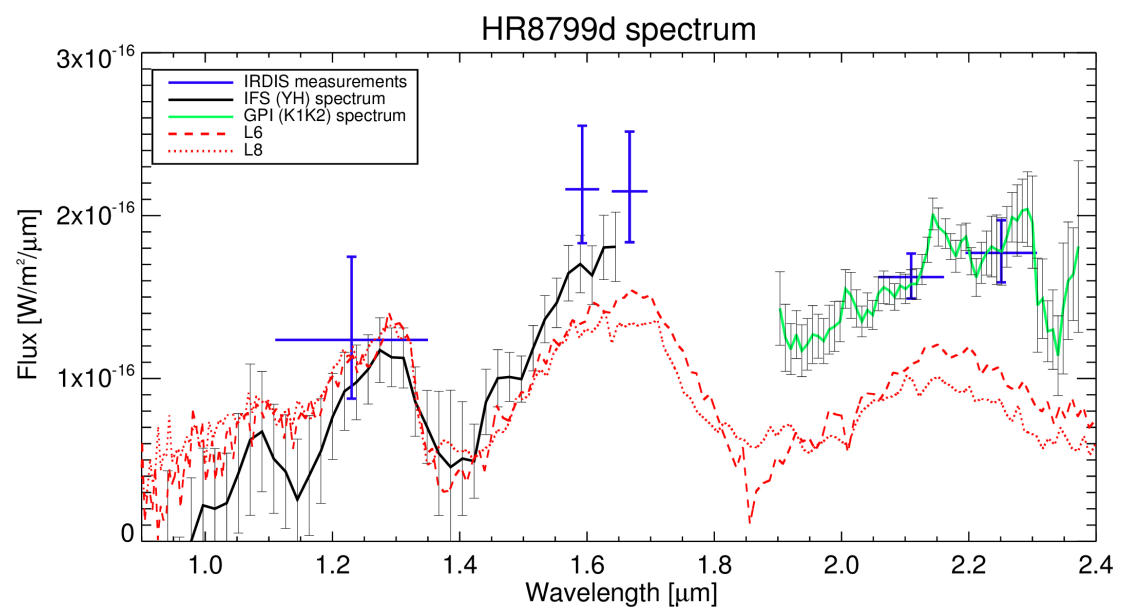

Fig. 10. Spectrum of planet $d$ as extracted from IFS data. IRDIS photometric points are overplotted. The GPI spectrum is also included in the plot. Comparison with a L6-L8 isolated brown dwarf shows a mismatch at $K$-band wavelengths. There is a slight mismatch between IRDIS and IFS measurements, as we normalized separately the flux of the two detectors with the corresponding PSF, the offset could be induced by another variable.

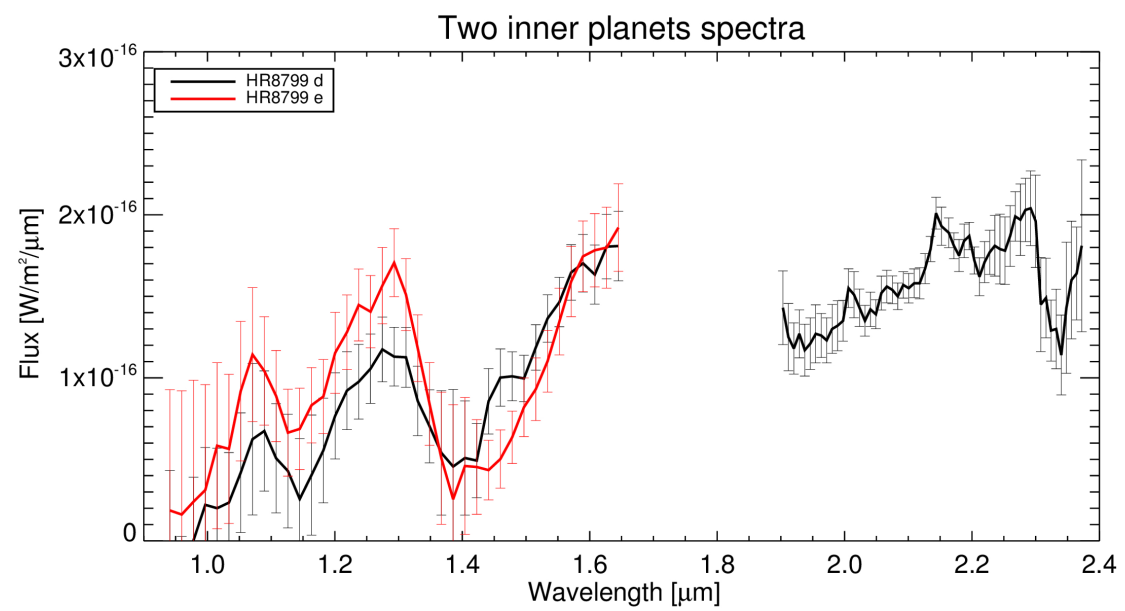

Fig. 11. Spectra of the two planets HR 8799 de together.

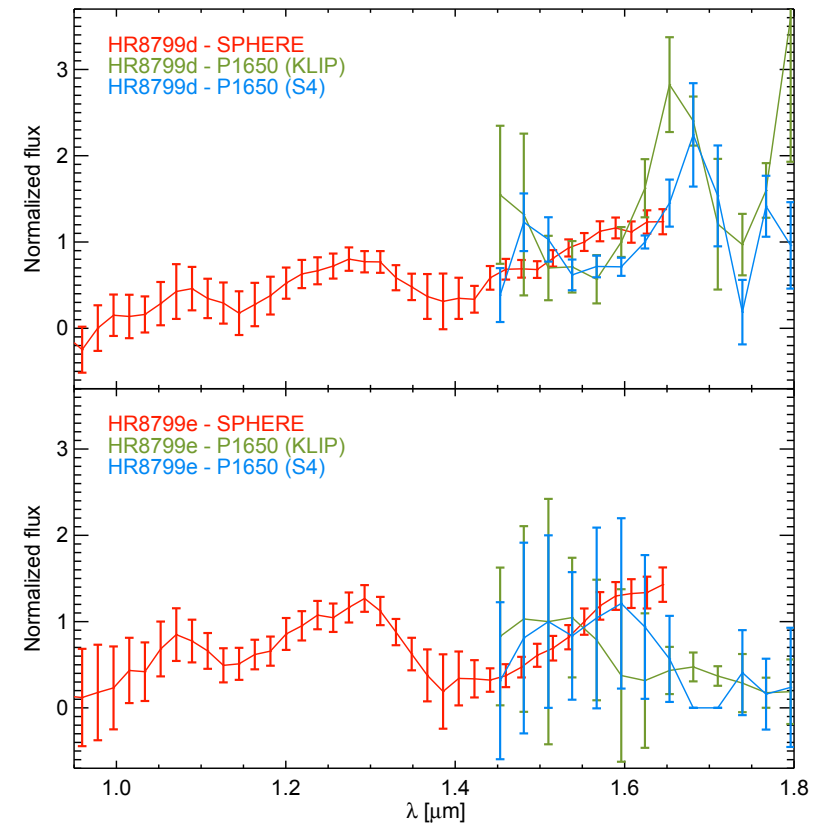

Fig. 12. Comparison of HR 8799 de spectra retrieved from SPHERE data and P1640 data (Oppenheimer et al. 2013). of July, August, and December 2014. Four previously known planets have been detected in $J, H 2 H 3, H$, and $K 1 K 2$ bands with high $\mathrm{S} / \mathrm{N}$ (reaching $\sim 200$ in $K$ band). For the first time, planet HR 8799 e has been detected in $J$ band. Optimized postprocessing methods were exploited in the analysis of these data. We used independent pipelines based on KLIP and T-LOCI, with the injection of fake negative companions to have robust measurements of photometry, spectrometry, and astrometry. We obtained photometry in the four dual- and broadband filters as well as spectra in $Y H$ band for HR 8799 de. The quality of these spectra, with mean $S / N \sim 20$, make them the highest quality spectra yet obtained for these objects.

The astrometric positions of the four planets are given for the epochs of July and December. For the closest planets d and e, we also provide astrometric positions of August 2014, which were extracted from IFS data. For this epoch, given the high $\mathrm{S} / \mathrm{N}$ ratio and the small pixel scale of IFS, we are able to obtain astrometric positions with error bars of 2-4 mas.

We find that two orbital solutions are the most consistent with our measurements for the two inner planets, corresponding to the resonance $2 \mathrm{~d}: 1 \mathrm{e}$ and $3 \mathrm{~d}: 2 \mathrm{e}$.

We found that a possible inner planet $\mathrm{f}$, which is expected to lie on the 3e:1f and 2e:1f resonance orbits, would be detected by IFS with masses greater than 3-7 $M_{\text {Jup }}$. Following 
previous stability simulations, we estimated that for the $3 \mathrm{e}: 1 \mathrm{f}$ orbit $2-4.5 M_{\text {Jup }}$ planets are not excluded, and in the $2 \mathrm{e}: 1 \mathrm{f}$ orbit we do not exclude the presence of 1.5-3 $M_{\text {Jup }}$ planets.

Finally, we demonstrate that the spectrophotometric properties of HR $8799 \mathrm{~d}$ and e in the $Y H$ range are similar to those of brown dwarfs with spectral type L6-L8. These planets show redder colors than field objects in the L-to-T transition. Empirical spectra of old brown dwarfs cannot describe the SED of these objects at longer wavelengths, and to comprehend the mechanisms that cause this red excess, further analysis is needed.

Acknowledgements. We acknowledge the two anonymous referees for their constructive comments. We are grateful to the SPHERE team and all the people at Paranal for the great effort during SPHERE commissioning runs. We especially thank A. Bellini and J. Anderson for the HST astrometric measurements. A.Z. acknowledges support from the Millennium Science Initiative (Chilean Ministry of Economy), through grant "Nucleus RC130007". D.M., A.-L.M., R.G., R.U.C., S.D. acknowledge support from the "Progetti Premiali" funding scheme of the Italian Ministry of Education, University, and Research. We acknowledge support from the French National Research Agency (ANR) through the GUEPARD project grant ANR10-BLANC0504-01 and the Programmes Nationaux de Planétologie et de Physique Stellaire (PNP and PNPS). Part of this work has been carried out within the frame of the National Centre for Competence in Research PlanetS supported by the Swiss National Science Foundation. S.P.Q. and M.R.M. acknowledge the financial support of the SNSF. The author thanks Sebastián Peréz for his help in solving unsolvable latex problems. SPHERE is an instrument designed and built by a consortium consisting of IPAG (Grenoble, France), MPIA (Heidelberg, Germany), LAM (Marseille, France), LESIA (Paris, France), Laboratoire Lagrange (Nice, France), INAF Osservatorio di Padova (Italy), Observatoire de Genève (Switzerland), ETH Zurich (Switzerland), NOVA (Netherlands), ONERA (France), and ASTRON (Netherlands), in collaboration with ESO. SPHERE was funded by ESO, with additional contributions from CNRS (France), MPIA (Germany), INAF (Italy), FINES (Switzerland), and NOVA (Netherlands). SPHERE also received funding from the European Commission Sixth and Seventh Framework Programmes as part of the Optical Infrared Coordination Network for Astronomy (OPTICON) under grant number RII3-Ct-2004-001566 for FP6 (2004-2008), grant number 226604 for FP7 (2009-2012) and grant number 312430 for FP7 (2013-2016).

\section{References}

Allard, F., Homeier, D., Freytag, B., \& Sharp, C. M. 2012, in EAS Pub. Ser. 57, eds. C. Reylé, C. Charbonnel, \& M. Schultheis, 3

Apai, D., Radigan, J., Buenzli, E., et al. 2013, ApJ, 768, 121

Baines, E. K., White, R. J., Huber, D., et al. 2012, ApJ, 761, 57

Barman, T. S., Macintosh, B., Konopacky, Q. M., \& Marois, C. 2011, ApJ, 733, 65

Barman, T. S., Konopacky, Q. M., Macintosh, B., \& Marois, C. 2015, ApJ, 804, 61

Bellini, A., Anderson, J., van der Marel, R. P., et al. 2014, ApJ, 797, 115

Bergfors, C., Brandner, W., Janson, M., Köhler, R., \& Henning, T. 2011, A\&A, 528, A134

Beuzit, J.-L., Feldt, M., Dohlen, K., et al. 2008, in SPIE Conf. Ser., 7014, 18 Boccaletti, A., Abe, L., Baudrand, J., et al. 2008, in SPIE Conf. Ser., 7015

Bohlin, R. C. 2007, in The Future of Photometric, Spectrophotometric and

Polarimetric Standardization, ed. C. Sterken, ASP Conf. Ser., 364, 315

Bonnefoy, M., Lagrange, A.-M., Boccaletti, A., et al. 2011, A\&A, 528, L15

Bonnefoy, M., Zurlo, A., Baudino, J. L., et al. 2016, A\&A, 587, A58

Bowler, B. P., Liu, M. C., Dupuy, T. J., \& Cushing, M. C. 2010, ApJ, 723, 850

Buenzli, E., Saumon, D., Marley, M. S., et al. 2015, ApJ, 798, 127

Burgasser, A. J., Cruz, K. L., Cushing, M., et al. 2010, ApJ, 710, 1142

Carbillet, M., Bendjoya, P., Abe, L., et al. 2011, Exp. Astron., 30, 39

Castelli, F., \& Kurucz, R. L. 2004, IAU Symp., 210, poster A20

Chauvin, G., Lagrange, A.-M., Beust, H., et al. 2012, A\&A, 542, A41

Chiu, K., Fan, X., Leggett, S. K., et al. 2006, AJ, 131, 2722

Claudi, R. U., Turatto, M., Gratton, R. G., et al. 2008, in SPIE Conf. Ser., 7014, $70143 \mathrm{E}$

Cowley, A., Cowley, C., Jaschek, M., \& Jaschek, C. 1969, AJ, 74, 375

Currie, T., Burrows, A., Itoh, Y., et al. 2011, ApJ, 729, 128

Currie, T., Fukagawa, M., Thalmann, C., Matsumura, S., \& Plavchan, P. 2012, ApJ, 755, L34

Currie, T., Burrows, A., Girard, J. H., et al. 2014, ApJ, 795, 133

Cutri, R. M., Skrutskie, M. F., van Dyk, S., et al. 2003, 2MASS All Sky Catalog of point sources

Cutri, R. M., et al. 2012, VizieR Online Data Catalog: II/311
Dahn, C. C., Harris, H. C., Vrba, F. J., et al. 2002, AJ, 124, 1170

Dohlen, K., Langlois, M., Saisse, M., et al. 2008, in SPIE Conf. Ser., 7014, 70143L

Esposito, S., Mesa, D., Skemer, A., et al. 2013, A\&A, 549, A52

Fukagawa, M., Itoh, Y., Tamura, M., et al. 2009, ApJ, 696, L1

Fusco, T., Rousset, G., Sauvage, J.-F., et al. 2006, Opt. Express, 14, 7515

Gagné, J., Lafrenière, D., Doyon, R., Malo, L., \& Artigau, É. 2014, ApJ, 783, 121

Galicher, R., Marois, C., Macintosh, B., Barman, T., \& Konopacky, Q. 2011, ApJ, 739, L41

Goździewski, K., \& Migaszewski, C. 2014, MNRAS, 440, 3140

Gray, R. O., \& Kaye, A. B. 1999, AJ, 118, 2993

Hinkley, S., Carpenter, J. M., Ireland, M. J., \& Kraus, A. L. 2011a, ApJ, 730, L21

Hinkley, S., Oppenheimer, B. R., Zimmerman, N., et al. 2011b, PASP, 123, 74

Hinz, P. M., Rodigas, T. J., Kenworthy, M. A., et al. 2010, ApJ, 716, 417

Høg, E., Fabricius, C., Makarov, V. V., et al. 2000, A\&A, 355, L27

Hughes, A. M., Wilner, D. J., Andrews, S. M., et al. 2011, ApJ, 740, 38

Hugot, E., Ferrari, M., El Hadi, K., et al. 2012, A\&A, 538, A139

Ingraham, P., Marley, M. S., Saumon, D., et al. 2014, ApJ, 794, L15

Janson, M., Bergfors, C., Goto, M., Brandner, W., \& Lafrenière, D. 2010, ApJ, 710, L35

Jones, A., Noll, S., Kausch, W., Szyszka, C., \& Kimeswenger, S. 2013, A\&A, 560, A91

Konopacky, Q. M., Barman, T. S., Macintosh, B. A., \& Marois, C. 2013, Science, 339,1398

Lafrenière, D., Marois, C., Doyon, R., \& Barman, T. 2009, ApJ, 694, L148 Lagrange, A.-M., Bonnefoy, M., Chauvin, G., et al. 2010, Science, 329, 57 Larkin, J., Barczys, M., Krabbe, A., et al. 2006, in SPIE Conf. Ser., 6269, 1 Looper, D. L., Kirkpatrick, J. D., Cutri, R. M., et al. 2008, ApJ, 686, 528 Macintosh, B., Graham, J. R., Ingraham, P., et al. 2014, PNAS, 111, 12661 Madhusudhan, N., Burrows, A., \& Currie, T. 2011, ApJ, 737, 34 Maire, A.-L., Boccaletti, A., Rameau, J., et al. 2014, A\&A, 566, A126 Maire, A.-L., Skemer, A. J., Hinz, P. M., et al. 2015, A\&A, 576, A133 Maire, A.-L., Bonnefoy, M., Ginski, C., et al. 2016, A\&A, 587, A56 Marley, M. S., Saumon, D., Cushing, M., et al. 2012, ApJ, 754, 135

Marois, C., Lafrenière, D., Doyon, R., Macintosh, B., \& Nadeau, D. 2006a, ApJ, 641, 556

Marois, C., Lafrenière, D., Macintosh, B., \& Doyon, R. 2006b, ApJ, 647, 612

Marois, C., Macintosh, B., Barman, T., et al. 2008, Science, 322, 1348

Marois, C., Macintosh, B., \& Véran, J.-P. 2010a, in SPIE Conf. Ser., 7736, 1

Marois, C., Zuckerman, B., Konopacky, Q. M., Macintosh, B., \& Barman, T. 2010b, Nature, 468, 1080

Marois, C., Correia, C., Véran, J.-P., \& Currie, T. 2014, in IAU Symp. 299, eds. M. Booth, B. C. Matthews, \& J. R. Graham, 48

Matthews, B., Kennedy, G., Sibthorpe, B., et al. 2014, ApJ, 780, 97

McElwain, M. W., Brandt, T. D., Janson, M., et al. 2012, in SPIE Conf. Ser., 8446,9

Mesa, D., Gratton, R., Zurlo, A., et al. 2015, A\&A, 576, A121

Moór, A., Ábrahám, P., Derekas, A., et al. 2006, ApJ, 644, 525

Morley, C. V., Fortney, J. J., Marley, M. S., et al. 2012, ApJ, 756, 172

Noll, S., Kausch, W., Barden, M., et al. 2012, A\&A, 543, A92

Oppenheimer, B. R., Baranec, C., Beichman, C., et al. 2013, ApJ, 768, 24

Pavlov, A., Feldt, M., \& Henning, T. 2008, in Astronomical Data Analysis Software and Systems XVII, eds. R. W. Argyle, P. S. Bunclark, \& J. R. Lewis, ASP Conf. Ser., 394, 581

Petit, C., Sauvage, J.-F., Fusco, T., et al. 2014, in SPIE Conf. Ser., 9148

Pueyo, L., Soummer, R., Hoffmann, J., et al. 2015, ApJ, 803, 31

Racine, R., Walker, G. A. H., Nadeau, D., Doyon, R., \& Marois, C. 1999, PASP, 111,587

Serabyn, G., Mawet, D., \& Burruss, R. 2010, BAAS, 42, 587

Sivaramakrishnan, A., \& Oppenheimer, B. R. 2006, ApJ, 647, 620

Skemer, A. J., Hinz, P. M., Esposito, S., et al. 2012, ApJ, 753, 14

Skemer, A. J., Marley, M. S., Hinz, P. M., et al. 2014, ApJ, 792, 17

Soummer, R. 2005, ApJ, 618, L161

Soummer, R., Brendan Hagan, J., Pueyo, L., et al. 2011, ApJ, 741, 55

Soummer, R., Pueyo, L., \& Larkin, J. 2012, ApJ, 755, L28

Spiegel, D. S., \& Burrows, A. 2012, ApJ, 745, 174

Stephens, D. C., Leggett, S. K., Cushing, M. C., et al. 2009, ApJ, 702, 154

Stetson, P. B. 1987, PASP, 99, 191

Su, K. Y. L., Rieke, G. H., Stapelfeldt, K. R., et al. 2009, ApJ, 705, 314

Sudol, J. J., \& Haghighipour, N. 2012, ApJ, 755, 38

Testi, L., D'Antona, F., Ghinassi, F., et al. 2001, ApJ, 552, L147

Thalmann, C., Schmid, H. M., Boccaletti, A., et al. 2008, in SPIE Conf. Ser., 7014

van Leeuwen, F. 2007, A\&A, 474, 653

Vigan, A., Moutou, C., Langlois, M., et al. 2010, MNRAS, 407, 71 
Vigan, A., N'Diaye, M., Dohlen, K. (SPHERE Consortium) 2016, A\&A, in press, DOI: 10.1051/0004-6361/201527584

Zuckerman, B., Rhee, J. H., Song, I., \& Bessell, M. S. 2011, ApJ, 732, 61

Zurlo, A., Vigan, A., Mesa, D., et al. 2014, A\&A, 572, A85

1 Núcleo de Astronomía, Facultad de Ingeniería, Universidad Diego Portales, Av. Ejercito 441, Santiago, Chile

e-mail: alice.zurlo@mail.udp.cl

2 Departamento de Astronomía, Universidad de Chile, Casilla 36-D, Santiago, Chile

3 Aix-Marseille Université, CNRS, LAM (Laboratoire d'Astrophysique de Marseille) UMR 7326, 13388 Marseille, France

4 INAF-Osservatorio Astronomico di Padova, Vicolo dell'Osservatorio 5, 35122 Padova, Italy

5 European Southern Observatory, Alonso de Cordova 3107, Vitacura, Santiago, Chile

${ }^{6}$ LESIA, Observatoire de Paris, CNRS, Université Pierre et Marie Curie - Paris 6 and Université Paris Diderot - Paris 7, 5 place Jules Janssen, 92190 Meudon, France

7 Université Grenoble Alpes, IPAG, 38000 Grenoble, France

8 CNRS, IPAG, 38000 Grenoble, France

9 European Southern Observatory, Karl-Schwarzschild-Strasse 2, 85748 Garching, Germany

${ }^{10}$ Laboratoire Lagrange, UMR 7293, Université de Nice SophiaAntipolis, CNRS, Observatoire de la Cote d'Azur, Bd. de l'Observatoire, 06304 Nice, France
11 Steward Observatory, Department of Astronomy, University of Arizona, 993 North Cherry Avenue, Tucson, AZ 85721, USA

12 Lunar and Planetary Laboratory, University of Arizona, 1640 E. Univ. Blvd., Tucson, USA

13 INAF - Astrophysical Observatory of Capodimonte, Salita Moiariello 16, 80131 Napoli, Italy

14 Anton Pannekoek Institute for Astronomy, University of Amsterdam, PO Box 94249, 1090 GE Amsterdam, The Netherlands

15 Max-Planck-Institut für Astronomie, Königstuhl 17, 69117 Heidelberg, Germany

16 Office National d'Etudes et de Recherches Aérospatiales, 29 avenue de la division Leclerc, 92322 Châtillon, France

17 Kiepenheuer-Institut für Sonnenphysik, Schöneckstr. 6, 79104 Freiburg, Germany

18 Stockholm University, AlbaNova University Center, Stockholm, Sweden

19 CRAL, UMR 5574, CNRS, Université Lyon 1, 9 avenue Charles André, 69561 Saint Genis Laval Cedex, France

20 Department of Astronomy, California Institute of Technology, 1200 E. California Blvd, MC 249-17, Pasadena, CA 91125 USA

21 Institute for Astronomy, ETH Zurich, Wolfgang-Pauli-Strasse 27, 8093 Zurich, Switzerland

22 Observatoire de Genève, University of Geneva, 51 Chemin des Maillettes, 1290 Versoix, Switzerland

23 INAF-Osservatorio Astrofisico di Arcetri, Largo E. Fermi 5, 50125 Firenze, Italy 